\title{
Le développement des utilisations non alimentaires de I'huile de colza dans I'UE : quels impacts sur les marchés et prix mondiaux ? ${ }^{1}$
}

\author{
Yves DRONNE \\ Alexandre GOHIN \\ INRA SAE2, rue Adolphe Bobierre, CS 61103, \\ F-35011 Rennes Cedex \\ $<$ Yves.Dronne@rennes.inra.fr> \\ $<$ Alexandre.Gohin@rennes.inra.fr>
}

\begin{abstract}
For some years, biodiesel production, based mainly on rapeseed oil, has registered a big progression, and important investments are on stream for the next years. The analysis of recent evolution of European and international rapeseed products suggests that this development has already significant effects in term of increase in cultivated area and crops, diminution of EU exportation positive balance for this seed and oil, and evolution of prices ratios for oils and meals. This hypothesis is confirmed by results obtained with OLEOSIM model simulating the effect of an exogenous shock of demand of this oil inside EU. Nevertheless the influence of other factors remains very strong, some of these factors are depending on the economic and climatic situation, some others are structural such as the withdrawal of EU from Canadian rapeseed export market. In a middle term situation, the prices spreads between rapeseed and other vegetable oils, due to the only biodiesel development should be weaker than during 2005 year.
\end{abstract}

Keywords: rapeseed oil, European Union, market

3,5 milliards de tonnes alors que celle des 17 principales huiles et graisses végétales et animales est voisine de 140 millions de tonnes soit moins de $4 \%$ de ce tonnage.

Les décisions des pouvoirs publics prises au niveau de l'UE et plus particulièrement par les gouvernements de la France et de l'Allemagne et les importants projets d'investissements industriels conduisent à envisager un fort développement de la production de biodiesel et donc de la demande non alimentaire de corps gras au cours des prochaines années. Dans un premier temps cette demande a été satisfaite pour l'essentiel par de l'huile de colza obtenue à partir de graines cultivées sur des surfaces mises en jachère dans le cadre de la politique agricole commune (PAC). Compte tenu des limites agronomiques aux surfaces disponibles pour les oléagineux dans ce cadre, la nouvelle réglementation communautaire a introduit en 2004 une aide spécifique aux cultures énergétiques (ACE) cultivées sur les surfaces en céréales, oléagineux et protéagineux (SCOP). Ceci implique (notamment pour le colza) une concurrence accrue entre graines à destination non alimentaire et graines à destination alimentaire dans l'affectation des surfaces SCOP de chaque pays.

L'analyse des évolutions passées de l'UE pour le marché du colza en termes de productions, utilisations et prix révèle un certain nombre de modifications importantes avec un fort développement de la production communautaire de biocarburants. Cependant différents facteurs autres que l'effet de la demande non alimentaire tels que les variations de rendement en colza au sein de l'UE, les capacités de trituration communautaires, les disponibilités à l'exportation en graines et en huiles de certains grands pays producteurs de colza, et de façon générale l'état de l'offre et de la demande mondiale en graines et huiles oléagineuses, ont certainement également eu un rôle significatif. Pour analyser le rôle spécifique de la demande non alimentaire en huile de colza dans I'UE, des simulations ont été réalisées avec le modèle Oléosim qui a été développé par l'Inra avec l'aide de I'Interprofession oléagineuse française.

Dans cet article, on présentera dans une première partie une analyse des évolutions enregistrées sur le marché communautaire du colza au cours des dernières campagnes en termes de productions et d'utilisations. Dans une seconde partie, nous rappelons des éléments de base sur les développements récents et prévus de la demande non alimentaire. Enfin, dans une troisième partie, nous analysons un certain nombre de résultats synthétiques obtenus par simulation à l'aide du modèle Oléosim qui permettent d'isoler le rôle de la demande non alimentaire sur les modifications du marché de cette huile, toutes choses étant égales par ailleurs.

\section{Un marché du colza en plein renouveau dans I'UE}

Ce renouveau du marché communautaire du colza s'est manifesté à la fois au niveau des productions et utilisations intérieures de graines, huiles (et tourteaux), mais aussi au niveau des évolutions des différents prix en valeur absolue et encore plus en valeur relative par rapport aux principaux produits concurrents.

\footnotetext{
Les auteurs remercient I'Interprofession oléagirecherche.
} 


\section{Des productions et utilisations en fort développement}

Le colza sous forme de graine, d'huile et de tourteau occupe traditionnellement une place importante en France, en Allemagne et dans I'UE, aussi bien au niveau de la production agricole que de la transformation (trituration) et des utilisations en alimentation animale (principalement tourteaux et à moindre titre graines entières et huiles), en alimentation humaine et dans certains secteurs non alimentaires (savonnerie, peintures, vernis, lipochimie, etc.) pour les huiles [1]. À toutes ces utilisations s'ajoute dorénavant celle pour les biocarburants.

Pour les 25 pays actuels de l'UE, la consommation totale de cette huile est passée de 4,1 millions de tonnes (Mt) en 2000/2001 à environ 5,3 en 2004/2005, soit une progression considérable de 1,2 Mt (+ $29 \%$ ) en seulement 4 ans. Pour le palme, avec un tonnage qui est passé de 2,8 Mt à 4,2 Mt au cours de la même période, on a une progression encore plus forte de 1,4 Mt (+ $50 \%)$. La très forte augmentation de l'utilisation communautaire de ces deux huiles contraste avec la quasi-stagnation de la plupart des autres huiles. Leur part dans la consommation totale communautaire est passée dans le même temps de 34 à $41 \%$, I'huile de colza demeurant de loin la première huile consommée dans I'UE. La progression de 2,6 Mt de la consommation de l'ensemble de ces produits est donc presque totalement imputable à ces deux seules huiles. Pour ces deux huiles la progression des utilisations observée au cours des dernières campagnes $s^{\prime}$ inscrit dans la continuité de la tendance enregistrée au cours des années 90 (+ 1,0 Mt en palme pour I'UE à 15 sur la décennie et $+1,6$ Mt pour le colza), mais il s'agit d'une très forte accélération du phénomène avec un rythme de progression qui a plus que doublé pour ces deux huiles.

Alors que pour le palme les utilisations intérieures restent totalement dépendantes des importations en provenance de quelques pays, essentiellement la Malaisie et I'Indonésie, pour I'huile de colza, la production issue de la trituration communautaire a toujours été et demeure l'élément essentiel pour l'approvisionnement de I'UE. Entre 1990 et 2000 la production intérieure de cette huile dans I'UE à 15 est passée de 2,7 à 3,8 Mt. Sur la décennie 90 les importations communautaires ont toujours été négligeables, mais les exportations vers les pays tiers ont culminé à 1,15 Mt en 1991 et étaient encore de 0,46 Mt en 2000. Durant cette période, la croissance de la consommation communautaire d'huile de colza $(+1,8 \mathrm{Mt})$ a été plus forte que celle de la production $(+1,1 \mathrm{Mt})$. Ceci a été rendu possible par une réduction des exportations vers les pays tiers qui à l'époque concernaient principalement la Chine, le Mexique, le Maroc et le Sénégal. La tendance à la baisse des exportations s'est poursuivie pour les 25 pays actuels de I'UE après 2000 avec un tonnage revenu à 100000 tonnes en 2005 (à destination principalement de l'Algérie), mais simultanément les importations se sont développées (prévisions de 250000 tonnes en 2005/2006 contre seulement 30000 tonnes au cours de la campagne précédente).

La progression de la production d'huile de colza dans l'UE s'est appuyée sur un développement de la trituration et de la production de graines dans les 25 pays actuels de l'UE (respectivement 12,9 et $15,3 \mathrm{Mt}$ en 2004/2005 contre 10,2 et 11,2 Mt en 2000/2001). Durant toute cette période, l'augmentation de la production communautaire de graines a donc été suffisante pour satisfaire les besoins croissants de la trituration et maintenir un certain courant d'exportation de graines vers les pays tiers.

Selon les estimations et prévisions actuelles de la revue Oil World [2], la situation du marché du colza pour la campagne en cours 2005/2006 et pour la prochaine campagne devraient faire apparaitre des évolutions extrêmement importantes.

$\mathrm{Au}$ niveau des surfaces cultivées en colza (figure 1), après avoir culminé à 4,8 millions d'hectares en 1999 (Mha), à la veille de la mise en place de l'Agenda 2000, celles-ci ont brutalement chuté à 4,0 Mha au cours de la campagne suivante avant de reprendre une modeste progression jusqu'en 2003 (4,1 Mha). En fait, c'est au cours des deux dernières années que la hausse des surfaces s'est réellement manifestée avec respectivement +350000 ha en 2004,
+200000 ha en 2005 et une prévision de +400000 ha pour 2006. Ainsi, il aura fallu attendre 2006 pour retrouver et légèrement dépasser avec 5,1 Mha le niveau de 1999.

Si pour la récolte 2006, les rendements sont satisfaisants (de l'ordre de 3,3t/ha), comme en 2004 et 2005, la récolte de l'UE à 25 pourrait alors atteindre près de $17 \mathrm{Mt}$, ce qui permettrait de dépasser le précédent record enregistré en 1999 (14,2 Mt) et marquerait une augmentation de 1,6 Mt par rapport à 2005. Cette augmentation des disponibilités intérieures devrait permettre une augmentation de la trituration. Cependant, pour atteindre le niveau de 14,1 Mt rendu nécessaire par la forte demande intérieure en huiles, I'UE devra aussi recourir à une légère baisse de ces exportations de graines vers les pays tiers et surtout à une très forte augmentation de ses importations de graines qui pourraient atteindre 450000 tonnes (contre seulement 180000 tonnes à l'exportation). En termes d'huile de colza, la demande intérieure devrait s'élever à 6,1 Mt (+ 800000 tonnes par rapport à la campagne précédente), ce qui impliquerait une nouvelle baisse des exportations vers les pays tiers et une certaine augmentation des importations.

Globalement, il apparaît donc que depuis 5 ans, après la très forte chute enregistrée en 2000, I'UE a connu une très forte progression de ses surfaces cultivées en colza ainsi que de sa production de graines, et encore plus de sa trituration et de ses utilisations de cette huile. Ceci a toutefois nécessité une réduction drastique de ses exportations de graines de colza vers les pays tiers (pour mémoire 1,2 Mt en 1999/2000) et une progression de ses importations. Malgré une tendance à la diminution

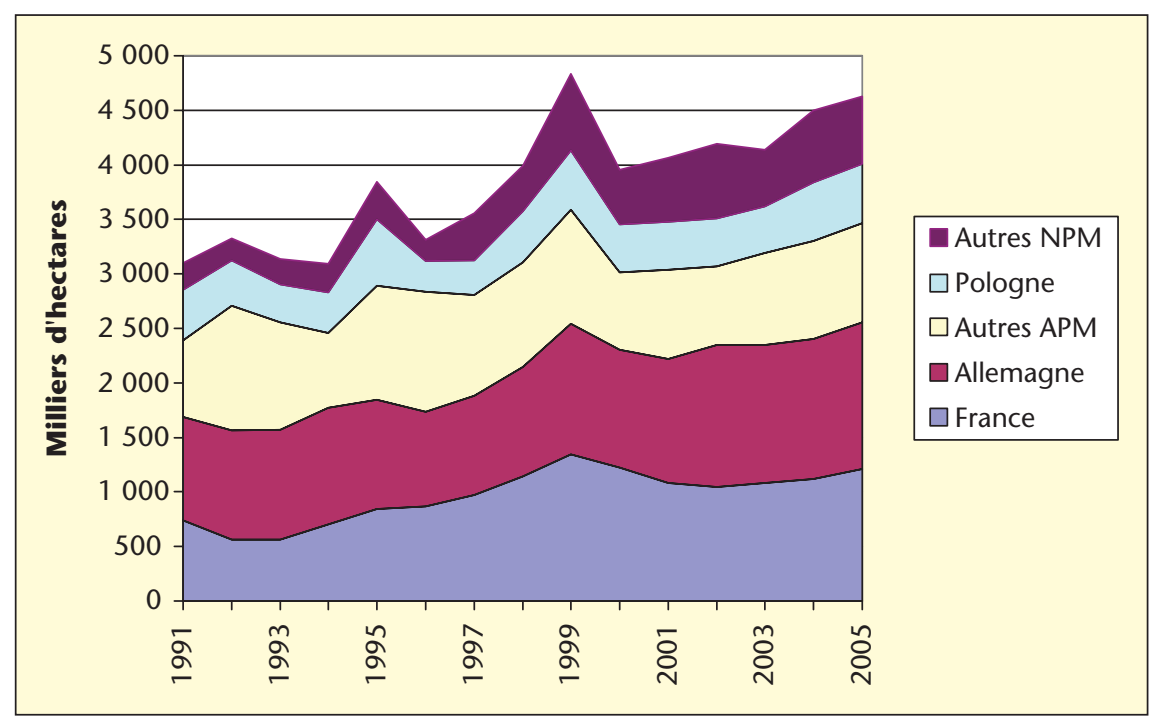

Figure 1. Évolution des surfaces en colza dans les 25 pays actuels de I'UE.

Unité : milliers d'hectares. Source : calculs à partir de Oil World. $A P M=15$ anciens pays de I'UE, NPM = 10 nouveaux pays de I'UE. 
des triturations communautaires de soja et de tournesol, les possibilités de transformation des graines de colza au sein de I'UE semblent actuellement atteindre un plafond dans l'attente de la reconversion de certaines usines fonctionnant au soja, voire de nouveaux investissements. Ceci explique la tendance nouvelle à l'importation directe d'huiles de colza. On peut donc déjà anticiper que la forte croissance de la demande de I'UE en huiles, notamment pour le biodiesel, a eu des effets internes importants en termes de surfaces cultivées et de niveaux de trituration, mais tend à affecter de plus en plus les grands équilibres du marché mondial du colza et même de l'ensemble des huiles végétales. Selon les données provisoires d'Oil World pour 2005/2006 [2], I'UE devrait non seulement être pour la première fois de son histoire déficitaire en huile de colza avec respectivement 250000 tonnes à l'importation et seulement 80000 à l'exportation, mais aussi devenir devant la Chine le premier importateur mondial d'huiles végétales.

\section{Des évolutions de prix de plus en plus différenciées par rapport aux autres produits du complexe oléagineux}

Les évolutions des prix communautaires des huiles et tourteaux de colza (et donc des graines) continuent naturellement à être fortement influencées par la conjoncture générale du marché mondial des oléagineux - et donc par les prix des trois produits leaders que sont le tourteau de soja, et les huiles de palme et de soja. Cependant, compte tenu du caractère actuellement très inélastique de la demande supplémentaire en huile de colza pour le biodiesel, les prix de deux coproduits de cette graine (huile et tourteau) tendent aujourd'hui à s'autonomiser.

La conjoncture s'est caractérisée au cours de l'année civile 2005, sous l'effet des augmentations de productions mondiales d'oléagineux très fortes pour la campagne 2004/2005 (environ $+46 \mathrm{Mt}$, soit environ $+14 \%$ ) et plus modérées pour la campagne 2005/2006 (environ +10 Mt soit $+2,7 \%$ ), par une baisse plus ou moins sensible de l'ensemble des prix du complexe oléagineux à commencer par la graine de soja US CAF Rotterdam qui est revenue de $307 \$ /$ t à $275 \$ / t$ (soit - 10,4\%), le tourteau de soja 48 Brésil CAF Rotterdam qui est revenu de $257 \$ / t$ à $233 \$ / t$ (soit - 9,3\%) et I'huile de soja Dutch FOB ex Mill qui est passée de $616 \$ /$ tà $545 \$ / t$ (soit-11,5\%). Pour l'huile de palme, avec une production mondiale qui a augmenté de 2,6 Mt en 2005 (soit + 8,4\%), on enregistre également une nette baisse du prix de ce produit ( $422 \$ / t$ contre 471 en 2004, soit $-10,4 \%$ ) pour le produit brut en CAF ports Nord Europe. Globalement (figures 2 et 3), on se trouve depuis quelques années dans

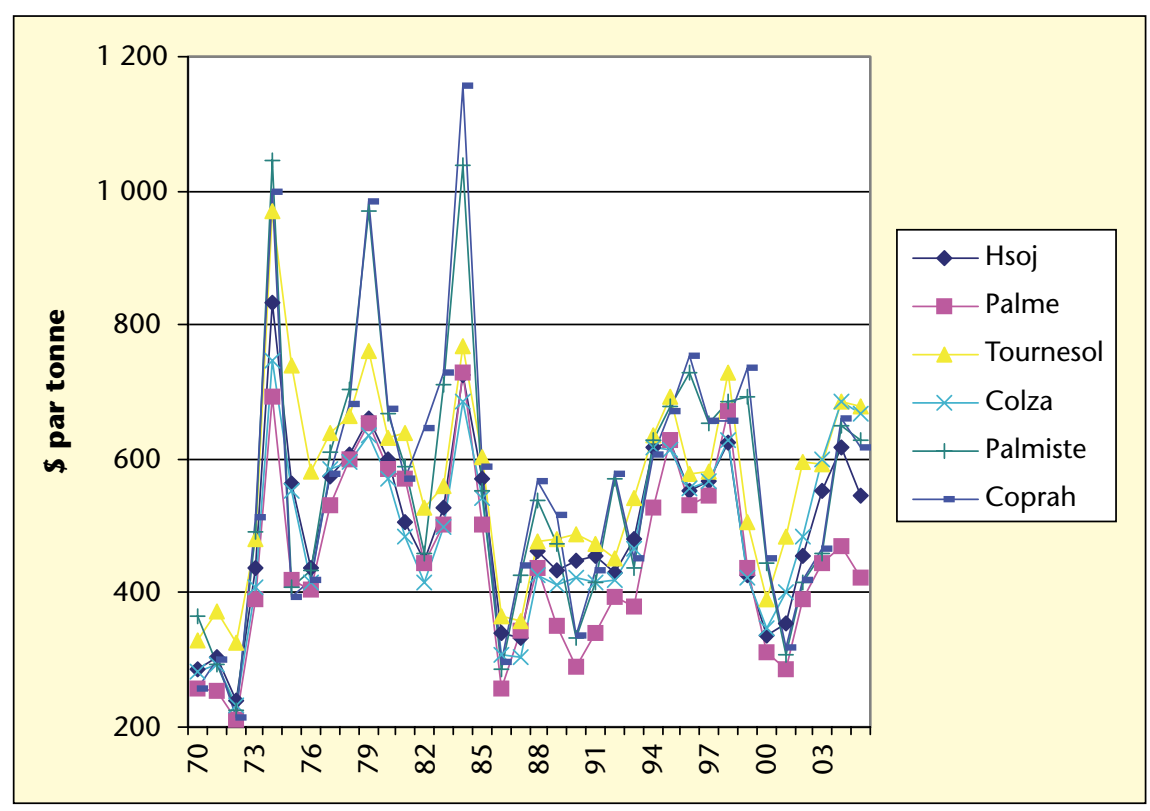

Figure 2. Évolution des prix internationaux des principales huiles. Unité : \$/tonne. Source: Oil World.

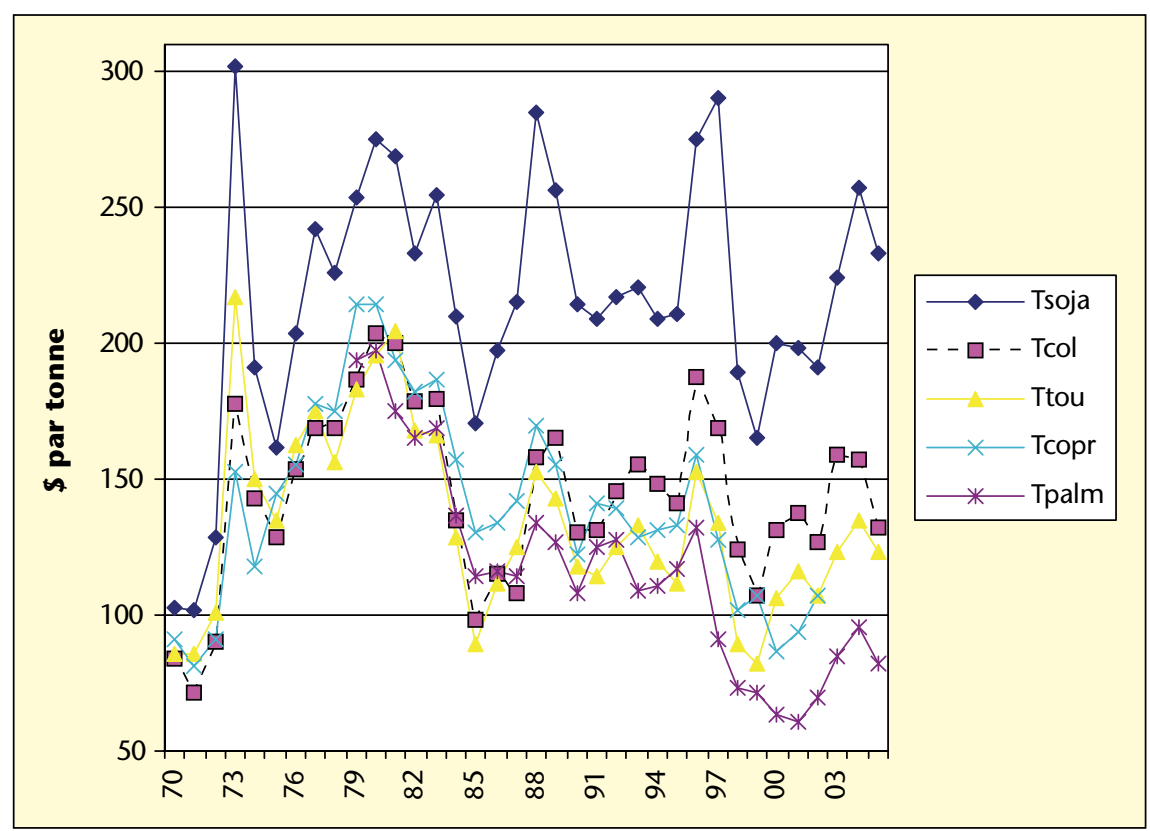

Figure 3. Évolution des prix internationaux des principaux tourteaux. Unité : \$/tonne. Source : Oil World.

une situation moyenne de prix pour le tourteau de soja (fourchette de 200 à 250 \$/t), de même que pour les principales huiles qui sont dans la fourchette 400 à $650 \$ / t$, niveaux bien inférieurs aux records enregistrés au cours des années 1974 à 1984

En fait ce qui caractérise actuellement plus particulièrement l'autonomie croissante du marché du colza par rapport aux autres oléagineux (et notamment au soja) est l'évolution de certains rapports de prix. Alors que durant les trente dernières années les rapports de prix (figures 4 et 5) entre les principales huiles fluides (soja, tournesol et colza) et les principaux tourteaux (soja, tournesol, colza) avaient tendance à évoluer de façon plus ou moins sensible en fonction principalement de facteurs liés à l'évolution conjoncturelle de l'offre, principalement au niveau communautaire, mais aussi en partie en fonction des conditions du marché 


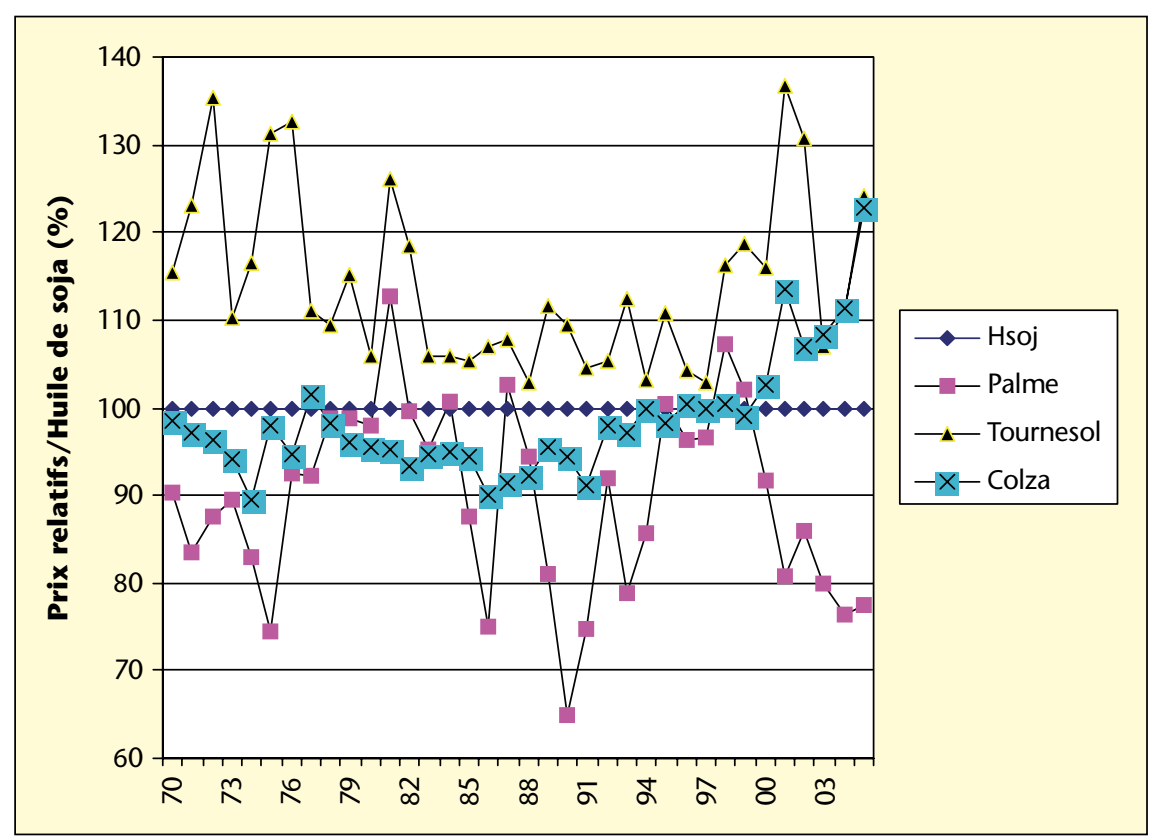

Figure 4. Évolution des prix relatifs des principales huiles par rapport au soja. Unité : pourcentages. Source : calculs à partir de Oil World.

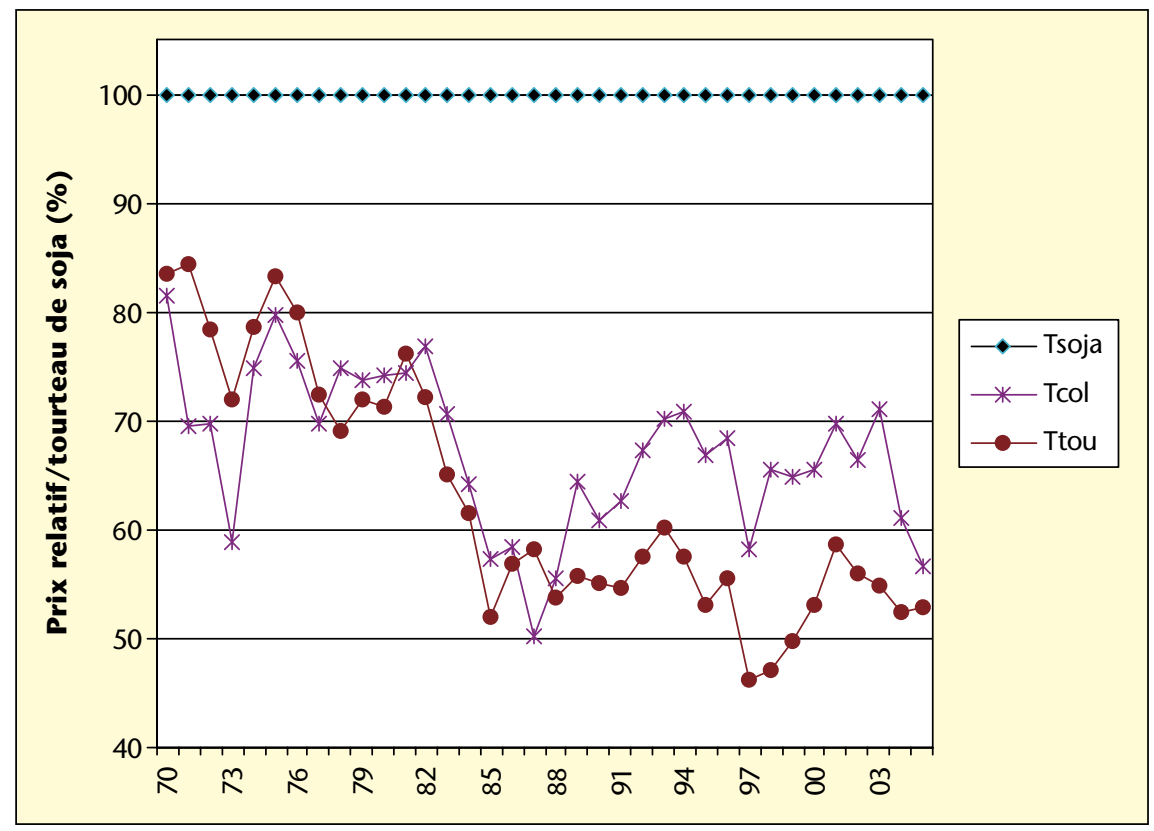

Figure 5. Évolution des prix relatifs des principaux tourteaux par rapport au soja. Unité : pourcentages. Source : calculs à partir de Oil World.

mondial (en particulier de la pression exercée par l'offre de graines du Canada, de l'Australie et plus récemment de certains pays d'Europe centrale et orientale), depuis le début des années 2000 c'est principalement au niveau de la demande qu'il faut rechercher les explications des fortes modifications des rapports de prix.

Pour I'huile de colza, un certain renchérissement par rapport au soja s'observe dès l'année au contraire en 2004 et 2005 une forte chute du rapport de prix par rapport au tourteau de soja qui s'expliquerait principalement par l'accélération de la trituration de colza dans I'UE et la mise sur le marché de fortes quantités de ce tourteau. Une certaine baisse des prix relatifs de ce produit a alors été nécessaire pour permettre un écoulement normal du produit. La très forte progression de la demande $d^{\prime}$ huile de colza dans l'UE et l'accroissement des écarts de prix par rapport aux autres huiles $(125 \$ / t$ par rapport au soja et même 250 par rapport au palme en moyenne sur 2005), sont aujourd'hui souvent expliqués par le développement de la demande non alimentaire dans I'UE. II convient donc dans une seconde partie d'examiner l'évolution récente de ce marché ainsi que quelques perspectives avant $d$ 'identifier dans la troisième partie la contribution effective de ce marché dans ces évolutions.

\section{Un développement du biodiesel qui s'accélère dans I'UE}

Même si la production communautaire d'éthanol et de dérivés tels que I'ETBE ${ }^{2}$ à usage énergétique a connu un développement notable au cours des dernières années, nous nous limiterons à analyser les évolutions du marché des biodiesels ou $\mathrm{EMHV}^{3}$ principalement produits jusqu'ici à partir d'huile de colza. Le tableau 1 résume l'évolution de cette production dans I'UE à 15 dont le tonnage est passé entre 1992 et 2000 de 5000 tonnes à 660000 tonnes avant une forte accélération qui a permis d'atteindre près de 2 Mt en 2004 [3]. Les chiffres définitifs pour 2005 ne sont pas encore connus mais devraient probablement faire apparaitre une nouvelle et forte progression des tonnages. La France, sous l'impulsion de I'interprofession oléagineuse, a été le leader sur ce marché dès le début des années 90 et a dominé la production communautaire jusqu'en 2000. Puis elle a été largement dépassée par l'Allemagne qui a produit en 2004 presque trois fois plus de ce biocarburant. À ces deux pays sont venus s'ajouter à partir de 2002 quelques autres pays de I'UE à 15 , principalement I'Italie (320000 tonnes en 2004), le Danemark (70 000 tonnes) et l'Autriche (57000 tonnes). Parmi les nouveaux pays membres, depuis 2003, certains se sont également lancés dans cette production, principalement la République tchèque (60 000 tonnes en 2004) et pour des tonnages plus modestes la Slovaquie et la Lituanie.

Dans son numéro du 7 octobre 2005, Oil World [2] a présenté une approche de bilan des huiles dant le nouveau et plus fort renchérissement, enregistré au cours des années 2003 à 2005, est selon les experts du secteur principalement dû au développement de la demande non alimentaire. Pour le tourteau de colza, on observe

\footnotetext{
2 Ethyl-tertio-butyl-éther.

${ }^{3}$ Ester méthylique d’huile végétale.
} 


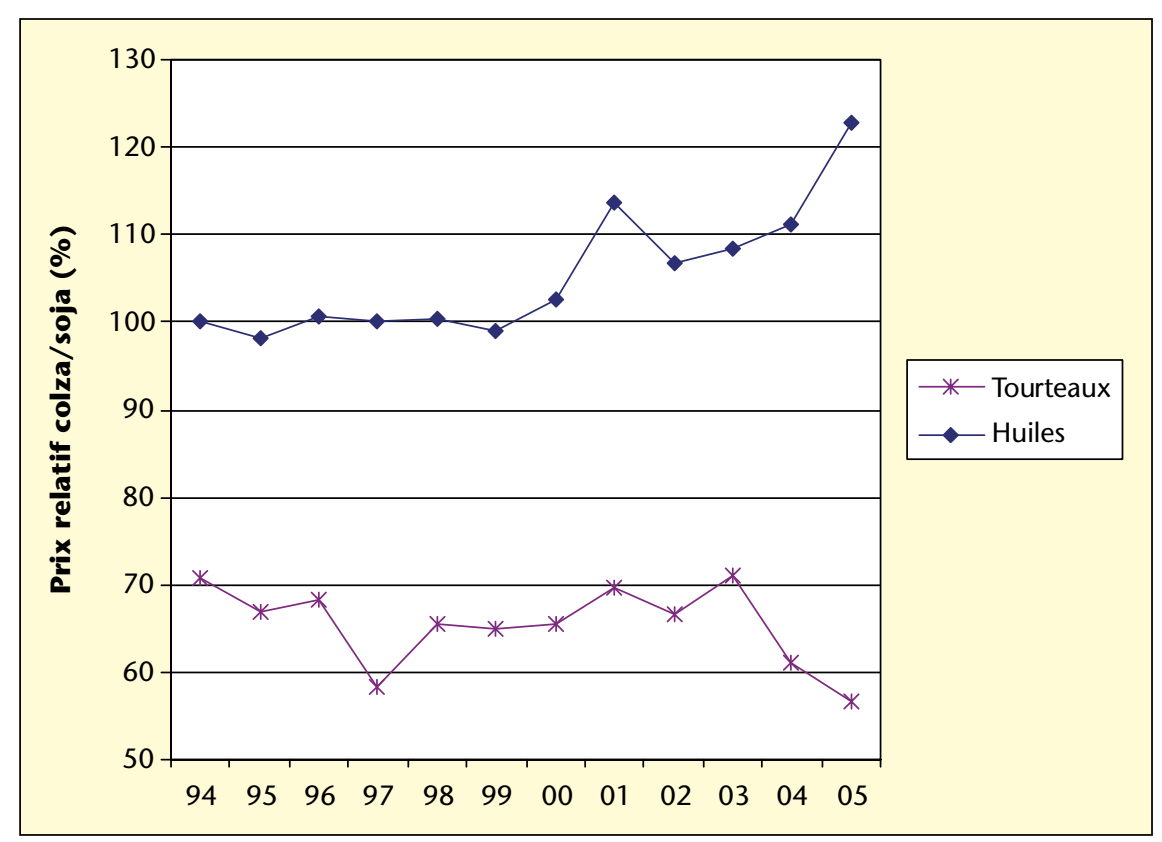

Figure 6. Évolution des ratios de prix colza/soja dans I'Union européenne au cours des dernières années pour les tourteaux et les huiles.

Unité : pourcentage. Source : calculs à partir de Oil World.

Tableau 1. Évolution de la production de biodiesel dans I'UE.

\begin{tabular}{|c|c|c|c|c|c|}
\hline & Allemagne & France & Autres & UE 15 & UE 25 \\
\hline 1992 & 5 & & & 5 & \\
\hline 1993 & 10 & 20 & & 30 & \\
\hline 1994 & 25 & 65 & & 90 & \\
\hline 1995 & 45 & 155 & & 200 & \\
\hline 1996 & 60 & 220 & & 280 & \\
\hline 1997 & 100 & 250 & & 350 & \\
\hline 1998 & 100 & 219 & & 319 & \\
\hline 1999 & 130 & 312 & & 442 & \\
\hline 2000 & 340 & 318 & & 658 & \\
\hline 2001 & 450 & 318 & & 768 & \\
\hline 2002 & 450 & 366 & 249 & 1065 & 1134 \\
\hline 2003 & 715 & 357 & 362 & 1434 & 1504 \\
\hline 2004 & 1035 & 348 & 461 & 1844 & 1933 \\
\hline
\end{tabular}

Unité : Milliers de tonnes. Source : Le Baromètre des biocarburants et European Biodiesel Board.

Tableau 2. Estimation de la répartition des utilisations d'huiles de colza dans I'Union européenne.

\begin{tabular}{|lccccc|}
\hline UE 25 & $\begin{array}{c}\text { Huile de } \\
\text { colza Total }\end{array}$ & $\begin{array}{c}\text { Dont } \\
\text { alimentaire }\end{array}$ & $\begin{array}{c}\text { Dont non } \\
\text { alimentaire }\end{array}$ & $\begin{array}{c}\text { Dont } \\
\text { biodiesel }\end{array}$ & $\begin{array}{c}\text { Dont utilisations } \\
\text { énergétiques directes }\end{array}$ \\
\hline $01 / 02$ & 4,00 & 2,88 & 1,12 & 1,12 & \\
$02 / 03$ & 4,14 & 2,69 & 1,45 & 1,45 & \\
$03 / 04$ & 4,38 & 2,60 & 1,78 & 1,74 & 0,01 \\
$04 / 05$ & 5,41 & 2,73 & 2,68 & 2,47 & 0,18 \\
$05 / 06 *$ & 6,02 & 2,64 & 3,38 & 3,07 & 0,30 \\
\hline
\end{tabular}

Source : Oil World. Unité : millions de tonnes ; * prévisions.

de colza au niveau de I'UE à 25 avec des prévisions pour 2005/2006. Il apparaît d'abord que si en 2004/2005 les utilisations dites « alimen- taires » (qui en fait incluent les utilisations en alimentation animale et certains usages industriels classiques hors énergie comme savonne- rie, peintures, vernis, lipochimie, etc.) représentaient encore à peu près $50 \%$ du total, leur place va se restreindre sensiblement dès 2005/2006 (à environ 44 \%) et que cette tendance observée depuis 2001 est probablement appelée à se poursuivre et s'accentuer. II apparaît ensuite qu'en tonnage, malgré la très forte hausse des utilisations totales communautaires $d^{\prime}$ huiles de colza (+ 2 Mt en 4 ans), les utilisations « alimentaires » de cette huile tendent à diminuer non seulement en part relative, mais aussi en tonnage du fait de certains reports de consommation sur d'autres huiles moins coûteuses telles que le palme, le soja ou le tournesol. Par ailleurs, au niveau des usages énergétiques, figure une estimation de 300000 tonnes d'huile de colza utilisée en l'état par les agriculteurs presque totalement en Allemagne (tableau 2).

Au début du développement de la production de biodiesel, l'approvisionnement des industries de I'UE s'est essentiellement effectué à partir des surfaces en colza cultivées sur jachère industrielle, donc hors SCOP $[4,5]$. Ces surfaces qui dépendent largement du taux de jachère obligatoire (en général $10 \%$, mais $5 \%$ en 2004), ont culminé à 850000 hectares en 1999 pour les pays de I'UE à 15 (tableau 3). Depuis 1996, la part des surfaces non alimentaires en colza a oscillé entre 20 et $25 \%$ pour I'UE à 15.

Même si toutes ces surfaces ne sont pas destinées au marché du biodiesel, on peut calculer une production «théorique » d'huile de colza en retenant un rendement forfaitaire de $42 \%$ en huile brute de la graine et en supposant que le rendement moyen en colza sur les surfaces en jachère est inférieur de $10 \%$ au rendement communautaire moyen de la campagne. Jusqu'en 2000, ces surfaces ont largement permis à elles seules de couvrir les besoins en huile de colza de l'industrie des biocarburants ainsi que de certains autres secteurs industriels (tableau 4). Pour rapprocher les utilisations $\mathrm{d}^{\prime}$ huile de colza par l'industrie du biodiesel des productions et utilisations globales de cette huile (traditionnellement exprimés en tonnage $\mathrm{d}^{\prime}$ huile brute), on a retenu (à titre indicatif) I'hypothèse que toute cette huile était issue de graines de colza, celles-ci ayant une teneur moyenne de $42 \%$ en huile brute et de $56 \%$ en tourteau, avec I'hypothèse d'un rendement de $96,5 \%$ au niveau du raffinage et de $97 \%$ de biodiesel pour une tonne d'huile raffinée au niveau de l'estérification. Avec ces hypothèses il faut donc 1,068 tonne d'huile brute pour obtenir 1 tonne de biodiesel. Le tableau 4 montre clairement, malgré les diverses approximations de calcul, que depuis 2002, les surfaces «non alimentaires » en colza, y compris les surfaces énergétiques sur SCOP depuis 2004, sont tout à fait insuffisantes pour couvrir 
Tableau 3. Évolution de la répartition des surfaces en colza dans I'UE à 15.

\begin{tabular}{|c|c|c|c|c|c|c|c|}
\hline UE 15 & $\begin{array}{c}\text { Surface totale } \\
\text { en colza }\end{array}$ & $\begin{array}{c}\text { Surface } \\
\text { en colza } \\
\text { « alimentaires » }\end{array}$ & $\begin{array}{l}\text { Part } \\
\text { des surfaces } \\
\text { alimentaires }\end{array}$ & $\begin{array}{c}\text { Surfaces } \\
\text { de colza } \\
\text { sur jachère }\end{array}$ & $\begin{array}{c}\text { Surfaces } \\
\text { de colza } \\
\text { " énergétique " }\end{array}$ & $\begin{array}{l}\text { Production totale } \\
\text { de graines } \\
\text { de colza }\end{array}$ & $\begin{array}{l}\text { Rendement moyen } \\
\text { toutes cultures } \\
\text { de colza }\end{array}$ \\
\hline 1996 & 2616 & 2055 & $80 \%$ & 561 & & 7338 & 2.81 \\
\hline 1997 & 2805 & 2494 & $89 \%$ & 311 & & 6730 & 2.40 \\
\hline 1998 & 3093 & 2746 & $89 \%$ & 347 & & 9524 & 3.08 \\
\hline 1999 & 3535 & 2683 & $76 \%$ & 852 & & 11403 & 3.23 \\
\hline 2000 & 2897 & 2146 & $75 \%$ & 751 & & 8950 & 3.09 \\
\hline 2001 & 2990 & 2316 & $78 \%$ & 674 & & 8870 & 2.97 \\
\hline 2002 & 3079 & 2334 & $76 \%$ & 745 & & 9501 & 3.09 \\
\hline 2003 & 3193 & 2454 & $77 \%$ & 739 & & 9507 & 2.98 \\
\hline 2004 & 3301 & 2628 & $80 \%$ & 464 & 209 & 11792 & 3.57 \\
\hline 2005 & 3465 & ND & ND & ND & ND & 12210 & 3.52 \\
\hline
\end{tabular}

Unités : milliers de tonnes et d’hectares. Source : calculs à partir de données Oil World, Cocéral et Proléa. ND : non disponible.

Tableau 4. Évolution des ressources et utilisations d'huile de colza dans I'UE à 15.

\begin{tabular}{|c|c|c|c|c|c|c|}
\hline & $\begin{array}{l}\text { Production potentielle } \\
\text { d'huile de colza } \\
\text { sur cultures non alimentaires }\end{array}$ & $\begin{array}{l}\text { Production } \\
\text { de biodiesel }\end{array}$ & $\begin{array}{c}\text { Utilisation } \\
\text { théorique d'huile } \\
\text { de colza }\end{array}$ & $\begin{array}{l}\text { Consommation } \\
\text { totale d'huile } \\
\text { de colza }\end{array}$ & $\begin{array}{l}\text { Consommation } \\
\text { en huiles } \\
\text { " alimentaires " }\end{array}$ & $\begin{array}{c}\text { Part des huiles } \\
\text { alimentaires dans } \\
\text { la consommation totale }\end{array}$ \\
\hline 1996 & 595 & 280 & 299 & 2252 & 1953 & $87 \%$ \\
\hline 1997 & 282 & 350 & 374 & 2453 & 2079 & $85 \%$ \\
\hline 1998 & 404 & 319 & 341 & 2750 & 2409 & $88 \%$ \\
\hline 1999 & 1039 & 442 & 472 & 3710 & 3238 & $87 \%$ \\
\hline 2000 & 877 & 658 & 703 & 3952 & 3249 & $82 \%$ \\
\hline 2001 & 756 & 768 & 820 & 4055 & 3235 & $80 \%$ \\
\hline 2002 & 869 & 1065 & 1137 & 4115 & 2978 & $72 \%$ \\
\hline 2003 & 832 & 1434 & 1532 & 4388 & 2856 & $65 \%$ \\
\hline 2004 & 909 & 1844 & 1969 & 5162 & 3193 & $62 \%$ \\
\hline 2005 & ND & ND & ND & ND & ND & ND \\
\hline
\end{tabular}

Unités : milliers de tonnes et d'hectares; Source : Calculs à partir de données Oil World, Cocéral et Proléa ; ND : non disponible.

I'ensemble des besoins de l'industrie du biodiesel et représenteraient moins de $50 \%$ en 2004. Ces besoins énergétiques qui représentent dès 2004 près de $38 \%$ des utilisations totales d'huile de colza de l'UE à 15 doivent donc être satisfaits de plus en plus par prélèvement sur les huiles «alimentaires» de colza (environ 900000 tonnes en 2004) et viennent donc en concurrence directe avec les usages traditionnels de ces huiles (embouteillage pour les consommateurs individuels, restauration collective, industries agricoles et alimentaires diverses). Cette concurrence directe entre débouchés traditionnels et nouveaux explique, au moins pour partie, l'augmentation du prix relatif de l'huile de colza.

En l'espace de 4 ans (de 2000 à 2004), la demande d'huile pour le biodiesel a donc augmenté d'environ 1,3 Mt dans I'UE à 15. Les perspectives de développement au cours des 5 prochaines années, si les objectifs sont atteints, sont en fait encore plus importantes. En effet, de très lourds investissements sont prévus dans de nombreux pays européens pour répondre d'ici 2010 à l'objectif de la Directive 2003/30/CE (qui fait suite à la publication du
«Livre Vert » de la Commission [6]) visant à promouvoir les différents biocarburants dont principalement le biodiesel (EMVH) et les dérivés d'éthanol (dont ETBE). L'objectif fixé est d'atteindre en 2010 un taux d'incorporation de ces biocarburants correspondant à 5,75\% de la valeur énergétique exprimée en pouvoir calorifique inférieur $(\mathrm{PCl})$ des carburants utilisés dans le secteur des transports contre $2 \%$ en $2005^{4}$.

Chaque pays de I'UE a jusqu'ici mis en œuvre des stratégies propres au niveau fiscal et industriel pour développer son marché en misant plus ou moins sur l'éthanol et ses dérivés ou sur les biodiesels. La France a mis en œuvre un système d'agréments des usines pour des tonnages fixés avec un programme important de développement des capacités de production dans le cadre d'abord de la première phase du plan national biocarburants (tableau 5) annon-

\footnotetext{
${ }^{4}$ Compte tenu des différences entre le $\mathrm{PCl}$ du gazole et celui du biodiesel ainsi que de leurs différences de densité, ceci correspond pour ce produit à un taux $d^{\prime}$ incorporation en tonnage d'environ 6,5\%.
}

cée en septembre 2004 par le Premier ministre. Dans le cadre de la seconde annonce de mai 2005, un supplément de capacité de 700000 tonnes est prévu pour la période 2008-2013. Selon le rapport sur l'« optimisation du dispositif de soutien à la filière biocarburants » publié en septembre 2005, la capacité agréée totale en tonnes de biodiesel devrait alors être de 2,47 millions de tonnes [7, 8].

Au niveau de l'ensemble de l'UE le rapport d'avril 2004 pour la Commission Bioenergy's role in the EU energie market: A view of developments until 2020 [9] prévoit pour 2010 une utilisation de 291 millions de tonnes d'équivalent pétrole (Mtep) pour I'UE à 15, 327 Mtep pour l'UE à 27 (y compris la Bulgarie et la Roumanie) incluant 65,3 Mtep pour l'Allemagne et 48,8 pour la France. En l'absence d'indication dans ce rapport des prévisions de répartition entre diesel et essence au niveau de l'UE et des différents pays, on a retenu une estimation dans tous les cas de $60 \%$ pour le diesel et $40 \%$ pour l'essence. On peut alors effectuer un calcul approximatif pour évaluer ce que représenterait en termes d'utilisation d'huile et de surface agricole la satisfaction de l'objectif de 
Tableau 5. Évolution des capacités d'estérification agréées en France.

\begin{tabular}{|lccc|}
\hline & \multicolumn{3}{c|}{ Plan biocarburants tranche 1 } \\
\cline { 2 - 4 } & 2004 & $2005-2006$ & $2007-2008$ \\
Grand Couronne & 260 & 260 & 260 \\
Compiègne & 83,5 & 100 & 100 \\
Boussens & 33 & 33 & 33 \\
Unités fonctionnelles & 376,5 & 393 & 393 \\
Sète & & 160 & 200 \\
Compiègne 2 & & & 100 \\
Montoir & & & 250 \\
Le Meriot & & 160 & 250 \\
Nouvelles unités & 376,5 & 553 & 800 \\
Total & & & 1193 \\
\hline
\end{tabular}

Unités : milliers de tonnes. Source : Saipol/Diester Industrie.
$5,75 \%$ en $\mathrm{PCl}$ (soit $6,5 \%$ en tonnage) en supposant que toute la demande est satisfaite par du biodiesel.

Les calculs théoriques (tableau 6) montrent d'abord que, pour I'UE à 15 , les besoins en surface de 8,75 Mha en 2010 ne pourront en aucun cas être satisfaits par la jachère communautaire qui en 2003, avec un taux de gel obligatoire de $10 \%$ hors petits producteurs, représentait environ 4 Mha pour la jachère obligatoire et 2,3 Mha pour la jachère volontaire. Seule une partie de ces terres est en fait utilisable pour des grandes cultures nonalimentaires (à titre indicatif moins de $50 \%$ en France) et, pour des raisons agronomiques, seule une partie de ces surfaces cultivées sur jachère peut en fait être consacré aux oléagineux. Dans son rapport Prospects for Agricultural Markets and Income (juillet 2005), la Commission européenne prévoit pour 2010 une superficie totale de 0,8 Mha de cultures non alimentaires totales sur un total de 8,2 Mha de jachères obligatoires et volontaires dans I'UE à 25 , soit un taux légèrement inférieur à $10 \%$. Le reste des besoins en oléagineux pour usages non alimentaires devra donc être trouvé sur des surfaces hors jachère de la SCOP estimées à 61,8 Mha en 2010 par le même rapport de la Commission, ces surfaces bénéficiant ou non de l'Aide aux cultures énergétiques (ACE) fixée actuellement à $45 € /$ ha, mais limitée à 1,5 Mha au niveau de I'UE. Selon l'estimation de surfaces en oléagineux de l'UE à 25 de la Commission [10] pour 2010 (6,3 Mha dont 3,9 pour le colza), on voit que le total des surfaces prévues en oléagineux ( $7,1 \mathrm{Mha})$ ne suffirait pas à elle seule à satisfaire les seuls besoins fixés pour le non-alimentaire, même si on suppose une poursuite de l'amélioration des rendements. Pour les surfaces en colza hors jachère, la Com-
Tableau 6. Évaluation des besoins en colza et surfaces agricoles à l'horizon 2010 pour satisfaire la Directive.

\begin{tabular}{|c|c|c|c|c|c|c|c|}
\hline & & Coeff. & Allemagne & France & Autres & UE 15 & UE 27 \\
\hline \multirow{2}{*}{$\begin{array}{l}\text { Consommation } \\
\text { de fuel }\end{array}$} & Mt & & 65,30 & 48,80 & 176,90 & 291,00 & 327,00 \\
\hline & & $60 \%$ & & & & & \\
\hline \multirow{2}{*}{$\begin{array}{l}\text { Consommation } \\
\text { de gazole }\end{array}$} & Mt & & 39,18 & 29,28 & 106,14 & 174,60 & 196,20 \\
\hline & & $6,5 \%$ & & & & & \\
\hline \multirow{2}{*}{$\begin{array}{l}\text { Besoins en } \\
\text { biodiesel }\end{array}$} & Mt & & 2,55 & 1,90 & 6,90 & 11,35 & 12,75 \\
\hline & & 1,068 & & & & & \\
\hline \multirow{2}{*}{$\begin{array}{l}\text { Besoins en huiles } \\
\text { brutes }\end{array}$} & Mt & & 2,72 & 2,03 & 7,37 & 12,12 & 13,62 \\
\hline & Rdt huile & $42 \%$ & & & & & \\
\hline \multirow{2}{*}{$\begin{array}{l}\text { Besoins en } \\
\text { graines }\end{array}$} & Mt & & 6,48 & 4,84 & 17,54 & 28,86 & 32,43 \\
\hline & $\mathrm{t} / \mathrm{ha}$ & 3,3 & & & & & \\
\hline $\begin{array}{l}\text { Besoins en } \\
\text { surface de colza }\end{array}$ & Mha & & 1,96 & 1,47 & 5,32 & 8,75 & 9,83 \\
\hline
\end{tabular}

Source : calculs personnels. Unités : millions de tonnes et millions d'hectares. mission ne prévoit en fait qu'une augmentation modes des surfaces (3,9 Mha en 2010 contre 3,5 en 2005 soit + $11 \%$ ).

Malgré le caractère très sommaire de ces prospectives et prévisions, il apparaît que pour satisfaire les objectifs de la Commission à l'horizon 2010 il faudrait théoriquement environ 10 Mt d'huiles végétales de plus qu'en 2004 pour le secteur du biodiesel dans la seule UE à 15. Les prévisions agricoles présentées par la Commission pour le secteur oléagineux à l'horizon 2010 sont très en retrait par rapport à ces objectifs. II apparaît donc intéressant d'analyser sur un plan théorique, à l'aide d'un modèle, ce que pourrait être pour l'UE et ses partenaires commerciaux, l'effet $d^{\prime}$ 'un choc exogène de demande non alimentaire se portant totalement sur I'huile de colza. En effet, globalement, dans l'analyse rétrospective des évolutions de prix et de rapport de prix, il n'est pas évident de pouvoir isoler et a fortiori quantifier le rôle des différents facteurs qui ont conduit à une situation donnée. C'est pourquoi il est indispensable de compléter les analyses par une modélisation.

\section{La modélisation de l'impact du développement du biodiesel dans I'UE}

Le développement des utilisations d'huiles non alimentaires dans I'UE peut s'analyser comme l'apparition d'un choc exogène sur la demande dans la mesure où pour des raisons fiscales et industrielles il s'agit d'une demande relativement captive sur une huile particulière (le colza) et que cette demande vient s'ajouter à la demande principalement alimentaire classique de cette huile. À ce niveau, les mécanismes antérieurs de substitution entre huiles en fonction des prix continuent à jouer de la part des consommateurs et des industriels de l'agroalimentaire. Les résultats présentés reposent sur I'utilisation du modèle Oléosim $[11,12]$ qui a été développé par l'Inra avec le concours financier de I'Interprofession oléagineuse (Proléa/ Fop). Les bases de la méthodologie retenue et les principales caractéristiques de ce modèle figurent dans les encadrés 1 et 2. Dans ce modèle mondial d'équilibre partiel multiproduits et multizones, compte tenu de l'importance des interrelations avec les céréales, aussi bien au niveau de la production agricole qu'au niveau de la demande en alimentation animale sont intégrés les marchés du blé, du maïs, de l'orge et de l'ensemble des autres céréales. Par ailleurs, au niveau de la demande pour chaque produit, on distingue une demande humaine, une demande animale et une demande industrielle. 


\section{Encadré 1. Quelques éléments de méthodologie}

Sur tout marché, le premier effet de l'apparition d'une demande exogène est dans un premier temps d'entraîner une hausse du prix de ce produit. Avec un délai plus ou moins long, la hausse de prix du produit entraîne d'une part une augmentation plus ou moins forte de l'offre de celui-ci et d'autre part une diminution de la demande des consommateurs. Ces deux phénomènes se poursuivant jusqu'à ce que le marché trouve un nouvel équilibre avec un prix plus élevé qu'avant le choc, une offre plus élevée et une demande « ajustable » c'est-à-dire susceptible de réagir aux effets de prix (avec possibilité de rationnement et le cas échéant de substitution partielle par d'autres produits) plus faible. C'est à la fois la hausse de la production et la baisse de la demande ajustable qui permettent de compenser exactement l'excès de demande exogène.

L'importance relative de la hausse de la production et de la baisse de demande ajustable dépend précisément des caractéristiques du marché du produit considéré et plus spécifiquement de paramètres que I'on appelle élasticités de l'offre et élasticités de la demande. Ces élasticités sont définies comme les variations en pourcentage d'une quantité (offre ou demande) en réponse à une variation de $1 \%$ d'un prix [13]. Dans des marchés tels que celui des huiles végétales (et de même pour les tourteaux) où il existe d'importantes possibilités de substitutions entre différents produits (huile de colza, huile de palme, huile de soja, etc.), on est amené à caractériser ces " possibilités » de substitutions entre produits par une matrice d'élasticité dans laquelle les coefficients de la diagonale représentent les élasticités directes (c'est-à-dire les variations en pourcentage de la demande d'un produit en réponse à une variation de $1 \%$ de son propre prix) et les autres coefficients représentent les élasticités croisées ( $c^{\prime}$ est-à-dire les variations en pourcentage de la demande d'un produit particulier quand le prix d'un autre produit varie de $1 \%)$. De façon générale, les élasticités directes de demande de tous les produits doivent être négatives (en vertu du principe « la demande baisse quand le prix monte ») et on appelle « produits substituables » deux produits qui ont une élasticité croisée négative, et produits complémentaires deux produits qui ont une élasticité croisée positive [14]. Au niveau de l'offre, le comporte-ment des producteurs peut se caractériser de la même façon dans le cas multiproduits par une matrice d'élasticités de l'offre prenant en compte les effets directs et croisés. La seule différence par rapport à la matrice de demande est que les élasticités directes doivent être positives en fonction du principe «l'offre augmente quand le prix monte ».

Une des particularités du marché des oléagineux est, en ce qui concerne les graines, de fournir deux produits liés (huile et tourteau) au niveau de la trituration dans des proportions pratiquement fixes qui dépendent essentiellement de la composition de ces graines et marginalement des technologies mises en œuvre. On est donc amené à analyser simultanément trois marchés qui sont celui des graines au niveau de l'offre et ceux des tourteaux et des huiles au niveau de la demande, les offres d'huiles et de tourteaux étant directement déterminées à partir de celles des quantités de graines triturées par ces coefficients considérés comme fixes de « rendements techniques moyens ».

Outre l'aspect multiproduits, une autre grande caractéristique du marché des oléagineux est son aspect multipays (ou multizone) qui correspond au fait que, d'une part, les huiles, les tourteaux et les graines sont largement échangés au niveau mondial et que, d'autre part, les niveaux de prix d'un même produit dans ces différentes zones sont liés entre eux. Cela ne veux pas dire que ces prix sont les mêmes mais seulement que toute variation de prix dans une zone se répercute de façon plus ou moins importante (et avec un certain délai) dans les autres, ces impacts sur les prix locaux dépendant notamment des instruments de politique agricole mis en œuvre (droits de douane, quotas, prélèvements/restitutions, etc.). Globalement l'équilibre des trois sous-marchés internationaux des oléagineux (graines, huiles, tourteaux) est assuré lorsque l'offre mondiale retrouve après le choc un niveau égalisant la demande mondiale de tourteaux et d'huiles). Cela revint à dire que pour chacun des produits considérés la somme des soldes du commerce extérieur des différentes zones est à nouveau nulle comme avant le choc.
Les deux simulations présentées correspondent à une hypothèse de choc de demande exogène de $3 \mathrm{Mt}$ d'huile de colza dans I'UE à 15. Par rapport à la situation de référence qui est constituée par l'année 2002, ceci correspondrait à un passage de la production communautaire de biodiesel de 1,1 Mt à 4,1 Mt, soit un quadruplement. Le chiffre retenu est bien sûr nettement inférieur à l'estimation les besoins pour couvrir l'objectif des $5,75 \%$ de la Directive communautaire à l'horizon 2010 (environ 11,4 Mt de biodiesel), mais correspond à peu près aux capacités industrielles qui devraient exister dans I'UE au début de I'année 2007. En tout état de cause, le niveau retenu pour l'accroissement de demande apparaît comme suffisant pour mesurer les principaux impacts sur les marchés internationaux.

\section{Effets du choc de demande avec transmission totale des prix}

\section{Des évolutions de prix différenciées selon les produits}

Le choc de demande d'huile de colza se manifeste d'abord par des modifications des différents prix communautaires. La hausse la plus importante est celle de l'huile de colza qui augmente de $19 \%$, conduisant par effet d'entraînement à des hausses de 9,6 à 11,1 \% des prix des autres huiles. En sens inverse, compte tenu de l'augmentation des mises sur le marché des différents tourteaux, les prix de ceux-ci diminuent 5,2\% pour le colza et de 0,6 à $3,5 \%$ pour les autres produits selon leur degré de substitution avec le tourteau de colza. Alors que la hausse du prix de l'huile de soja est voisine de celle de colza, la baisse du prix du tourteau de soja $(-0,63 \%)$ est beaucoup plus faible que celle du colza en raison d'un pourcentage de variation de l'offre beaucoup plus faible que pour les autres tourteaux. En $d^{\prime}$ 'autres termes l'impact d'une mise sur le marché d'un même supplément de tourteaux a beaucoup moins $d^{\prime}$ impact pour le soja qui correspond à un marché mondial de $131 \mathrm{Mt}$ que pour le colza qui correspond à un marché mondial de seulement 19 Mt. Les prix des graines oléagineuses enregistrent des changements différents les uns des autres, selon des modifications relatives des prix des différents tourteaux et huiles et selon leurs rendements respectifs en huiles et tourteaux. Alors que le prix de la graine de colza augmente de plus de $12 \%$ (effet principal huile), celle de soja ne 


\section{Encadré 2. Quelques caractéristiques du modèle Oléosim}

Le modèle Oléosim se présente globalement comme un modèle agricole sectoriel de statique comparative de structure analogue à des modèles tels que Swopsim [15] ou Miss qui ont été antérieurement largement utilisés pour des simulations de politique agricole [16]. Sa fonction essentielle est de permettre le calcul d'une nouvelle situation d'équilibre du marché mondial (productions, consommations, échanges, prix) en réponse à un ou plusieurs chocs exogènes, les nouveaux prix d'équilibres étant ceux qui permettent d'égaliser les nouvelles offres et demandes mondiales, ou, de façon équivalente, d'égaliser à zéro la somme des soldes nets des diverses zones géographiques pour les différents produits. Dans la mesure où la trituration constitue une opération industrielle intermédiaire permettant de mettre sur le marché des huiles et des tourteaux qui font l'objet de deux demandes spécifiques, nous n'avons pas cherché dans le cadre de ce travail à modéliser précisément ce processus et nous avons considéré (le cas du palme faisant l'objet d'une modélisation spécifique) que toute production de graine oléagineuse pouvait s'exprimer sous la forme d'une offre en tourteau et en huile sur la base des rendements techniques de chaque graine en tourteau et en huile. Le modèle n'aborde donc pas le problème de la localisation de la trituration mondiale. Par ailleurs, compte tenu du faible niveau moyen des marges de trituration, le prix de chaque graine a été exprimé comme somme pondérée des prix des tourteaux et huiles correspondants, les coefficients de pondération correspondant aux rendements techniques de chaque graine dans chaque zone. Ce sont donc ces prix pondérés qui interviennent dans le calcul des variations d'offre de chaque graine dans chaque zone.

Les comportements des producteurs agricoles par rapport à l'offre de graines d'oléagineux et de céréales sont résumés dans des matrices d'élasticités prix des surfaces et des rendements. De la même manière, les comportements des consommateurs (finaux et/ou intermédiaires) par rapport à la demande d'huiles, de tourteaux et de céréales sont captés dans des matrices d'élasticités prix de demande. Ces dernières reflètent bien évidemment les possibilités de substitution entre produits (par exemple, huiles végétales en alimentation humaine, tourteaux et céréales en alimentation animale). Seuls les échanges nets de graines et/ou produits sont représentés dans Oléosim. Cette hypothèse n'est pas très restrictive dans la mesure où Oléosim adopte une désagrégation fine des biens.

\section{Les produits}

Le modèle prend en compte les principaux oléagineux produits et échangés dans le monde : le soja, le colza et le tournesol mais aussi le palme qui prend une place de plus en plus importante, ainsi que l'arachide et le coprah. II existe une forte interaction entre les marchés des graines oléagineuses et ceux des huiles et des tourteaux. Des chocs sur les prix des graines oléagineuses par exemple se transmettent sur les marchés des huiles et des tourteaux. C'est pourquoi la modélisation ne peut se limiter aux graines, il est nécessaire de prendre en compte les huiles et les tourteaux. Enfin, il est important de modéliser aussi les marchés des principales céréales. En effet, il existe de fortes substitutions à l'offre entre les graines oléagineuses et les céréales, mais aussi à la demande dérivée entre les tourteaux et les céréales.

\section{Les céréales}

Les céréales sont généralement transformées avant d'être consommées, notamment pour I'alimentation humaine (farine, semoule, etc.). Toutefois, pour ne pas complexifier excessivement le modèle, nous n'avons pas modélisé les transformations des céréales, ce qui constitue d'ailleurs une pratique assez courante dans ce type de modèle. Nous avons pris en compte cinq catégories de céréales : le blé, le mais, l'orge, le riz, les céréales diverses. Les céréales diverses sont un agrégat de céréales moins importantes regroupant l'avoine, le millet, le sorgho et le seigle.

\section{Les oléagineux}

Les graines d'oléagineux sont une production intermédiaire : elles subissent une trituration servant à produire des huiles et des tourteaux. Les principales graines d'oléagineux ont été intégrées au modèle : le soja, le colza, le tournesol, le coprah, le palme et l'arachide. Chaque graine est triturée pour produire un seul type d'huile et un seul type de tourteau, hormis le palme qui présente des caractéristiques particulières. Le palme permet en effet la production de deux types d'huiles (I'huile de palme et I'huile de palmiste) et un type de tourteau (le tourteau de palmiste). Au total, sont représentées explicitement dans le modèle six graines et six tourteaux d'oléagineux et sept huiles végétales.Les productions prises en compte dans le modèle sont consommées pour satisfaire trois principaux types de demande :- la demande intermédiaire (ou animale) : cette demande est composée à la fois de céréales et de tourteaux;- la demande finale humaine : dans notre modèle la consommation humaine se compose de céréales et d'huiles ;- la demande finale non-alimentaire pour les huiles végétales.

\section{Les zones géographiques}

L'objectif principal d'Oléosim étant de simuler différents chocs sur le marché mondial des oléagineux, la désagrégation met naturellement en évidence les principaux acteurs sur ce marché. Nous avons bien évidemment distingué les États-Unis et l'UE. Nous avons divisé le reste du monde en plusieurs zones en fonction de leur rôle et leur importance dans les échanges internationaux de céréales et d'oléagineux. Au total, les différents pays/zones intégrés au modèle sont au nombre de sept. II s'agit de : - I'UE (Code UE) qui regroupe les quinze pays de I'UE. Ses principales productions sont pour les oléagineux le colza et le tournesol et pour les céréales le blé et l'orge.

- les Etats-Unis (code USA) : le soja et le maïs sont ses deux plus importantes cultures.

- la zone Argentine-Brésil (code ARG_BRE) qui, comme son nom l'indique, regroupe l'Argentine et le Brésil. Cette zone produit beaucoup de soja (environ $30 \%$ de la production mondiale).

- la zone Asie du Sud-Est (code ASIE_SE). Cette zone comprend I'Indonésie, la Malaisie et les Philippines et est une très grande productrice de palme et de coprah (respectivement $80 \%$ et $60 \%$ de la production mondiale).

- la zone Afrique-Asie (code AFR_ASI) qui comprend I'Inde, le Pakistan, I'Iran, le Bangladesh, l'Algérie, le Maroc, la Tunisie, l'Egypte et la Libye. II s'agit d'importants consommateurs d'oléagineux (surtout des huiles de soja, d'arachide, de palme et de tournesol ainsi que du tourteau d'arachide) et de céréales (de riz, de blé et de céréales diverses). Les principales productions de cette zone sont l'arachide (graines, huiles et tourteaux), le blé et le riz.

- la Chine (code CHINE). Nous avons distingué ce pays qui est à la fois un grand consommateur de céréales et d'oléagineux (elle représente environ $25 \%$ de la consommation humaine mondiale) et un grand producteur, notamment d'arachide, de colza et de riz.

- le reste du monde (code AUTRES). Cette dernière zone est définie par solde et est très hétérogène. La nécessité d'une désagrégation plus approfondie de ce dernier agrégat dépend naturellement des simulations envisagées. 
progresse que de 3,3\% (effet négatif du tourteau qui compense en partie l'effet huile).

$\mathrm{Au}$ niveau des prix communautaires, il est important d'analyser non seulement l'évolution en pourcentage des niveaux de prix, mais aussi l'évolution des rapports de prix en prenant pour références les prix de l'huile et du tourteau de soja. Pour les huiles, la seule modification importante des rapports de prix relatifs à l'huile de soja est le renchérissement de près de 8 points de l'huile de colza. Les autres rapports de prix évoluent peu à l'exception du prix de l'huile de tournesol qui diminue fortement par rapport au colza. Au niveau des tourteaux, les prix des tourteaux de colza et de tournesol baissent de respectivement 3 points et 1,5 point. Ces deux produits deviennent donc plus compétitifs et le colza voit son prix diminuer légèrement par rapport au tournesol.

Enfin, en ce qui concerne les céréales, on a retenu I'hypothèse que leurs prix restaient stables (effet stabilisant des prix d'intervention). On observe une légère baisse des prix relatifs de celles-ci par rapport à la graine de soja et à I'ensemble des oléagineux cultivés dans I'UE. De plus, le ratio de prix tourteau de soja sur blé qui concerne directement I'alimentation animale présente une légère diminution qui implique une légère diminution du rapport de prix « énergie » sur «protéine ». Enfin, pour les principales graines oléagineuses de l'UE (colza et tournesol) plus riches en huile, on a une amélioration du prix par rapport au soja.

\section{Les évolutions des prix et bilans au niveau mondial}

Le choc de demande simulé au sein de l'UE, a non seulement des effets sur les prix locaux mais aussi un certain nombre de conséquences au niveau international. Alors que les prix des céréales sont stables par hypothèse dans I'UE, les prix de ces produits au niveau international augmentent de 2,3 à 3,1\% du fait d'une légère diminution des surfaces mondiales cultivées en céréales. Ces pourcentages sont assez voisins de ceux enregistrés pour la graine de soja. Pour tous les autres produits (huiles, tourteaux et graines oléagineuses) puisqu'il y a transmission totale des prix, les variations de ceux-ci dans les différentes zones sont égales à celles constatées dans I'UE.

Au niveau des surfaces (à surface totale constante) on constate une diminution de celles en céréales d'environ 1,46 millions d'hectares (particulièrement marquée pour le blé, les céréales diverses et le maiis) et une progression de même niveau des oléagineux qui concerne principalement le colza ( $54 \%$ du total) et le tournesol (31\%). Ces deux fortes progressions sont liées à des augmentations des prix de ces graines (respectivement $12 \%$ et $7 \%$ ) nettement plus importantes que celles des prix des céréales (de l'ordre de $3 \%$ hors UE) et du soja $(3,3 \%)$. Bien que les prix du palme et $d u$ coprah soient en forte augmentation, l'effet sur les surfaces de ces cultures pérennes est très limité du fait de leurs faibles élasticités. En termes de production, ces variations de surface entraînent une diminution de $4,8 \%$ de la récolte de céréales (dont l'essentiel en blé et en maiis), et en sens inverse une augmentation des tonnages d'oléagineux (particulièrement en colza et tournesol).

Pour les différentes céréales, les diminutions de productions sont compensées par des baisses $d^{\prime}$ utilisation en alimentation humaine (environ - 3,2 Mt) du fait du renchérissement du prix et en alimentation animale $(-1,5 \mathrm{Mt}$ dont $-0,9$ Mt pour le seul maïs) du fait d'une concurrence accrue par les différents tourteaux et en particulier celui de soja. Dans la mesure où on ne prend pas en compte de variations de stocks, les hausses des productions de graines oléagineuses se traduisent par des augmentations des triturations, et donc des productions des huiles et tourteaux correspondants. En pourcentage des valeurs initiales, au niveau mondial, les modifications de productions et surfaces liées au choc de demande sont très faibles pour les céréales, de même que pour le palme et le coprah. Par contre, celles relatives aux graines de colza $(+3,5 \%$ en surface et $4,6 \%$ en production ${ }^{5}$ ) et à celles de tournesol $(+2,2 \%$ et $+2,5 \%)$ sont plus significatives.

En ce qui concerne les huiles, on a une augmentation de la production mondiale de 1,1 Mt (liée à l'augmentation des productions et triturations des diverses graines oléagineuses) qui est largement inférieure à l'augmentation de la demande en non alimentaire (3 Mt pour I'huile de colza dans I'UE). L'effet de « rationnement par les prix » se traduit par une diminution globale des utilisations humaines pour ces différentes huiles de 1,9 Mt. L'augmentation du prix de l'huile de colza étant nettement la plus forte, on a un net recul des utilisations alimentaires de cette huile $(-2,4 \mathrm{Mt}$ dans le monde), qui n'est que très partiellement compensée par des reports de consommation sur les autres huiles, notamment tournesol (+ 300000 tonnes) et palme (+ 100000 tonnes). Globalement on a donc un rationnement de la demande mondiale alimentaire d'huiles qui est de l'ordre de $2 \%$. Pour les tourteaux, dont les prix deviennent plus compétitifs, les utilisations en alimentation animale progressent de 1,5 Mt, principalement sous forme de colza ( $57 \%$ de la progression), mais

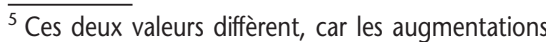
de surfaces ont lieu dans des zones où les rendements sont plus élevés que la moyenne mondiale.
}

aussi à un moindre titre sous forme de tournesol et de soja.

En valeurs relatives, une des plus fortes variations est enregistrée pour l'huile de colza, avec une augmentation de 4,7\% de la production et une baisse de $24 \%$ de la consommation humaine. Pour I'huile de tournesol, on a une augmentation de $3,4 \%$ de la production mondiale et de $4,3 \%$ des utilisations humaines, c'est donc le produit qui est le plus favorisé en terme de demande humaine, les progressions pour les autres produits, étant inférieure à 0,7 \% à l'exception du palmiste qui correspond à un tonnage relativement limité. Pour les tourteaux, les utilisations en alimentation animale progressent de 5,9\% pour le colza et de 3,5\% en tournesol, les autres produits n'enregistrant que de faibles variations, notamment le soja dont la consommation ne progresse que de $0,24 \%$.

\section{Les évolutions des bilans de I'UE}

À l'intérieur de l'UE, compte tenu de l'augmentation des prix des graines oléagineuses et de la stabilité de ceux des céréales, la surface de ces dernières recule de 340000 hectares (principalement en blé et orge) et augmente d'autant en oléagineux, essentiellement en colza et un peu en tournesol. Au niveau de la production, le recul est de 2,64 Mt en céréales ${ }^{6}$. Au niveau des utilisations, il n'y a pas de changement pour I'alimentation humaine (car les prix sont inchangés) et un très faible recul en alimentation animale (- 175000 tonnes) du fait d'une faible substitution par les tourteaux. Pour les oléagineux, la surface augmente de 340000 hectares essentiellement en colza et un peu en tournesol. L'augmentation de tonnage pour ces graines se porte totalement sur la trituration. En termes de solde du commerce extérieur de I'UE, l'excédent global en céréales diminue de 2,47 Mt.

En pourcentages, les évolutions les plus importantes sont celles de colza $(+8,8 \%$ en surface et production) et en tournesol $(+4,4 \%)$. Pour les céréales (hors riz), les reculs de surfaces vont de $1 \%$ à pratiquement $2 \%$. En termes de solde, les excédents diminuent fortement pour le blé (- 34\%), l'orge (-15\%) et pour les céréales diverses $(-14 \%)$. Pour le maïs, les importations nettes augmentent de $10 \%$. Pour toutes les huiles, les excédents sont diminués ou les déficits augmentés. Pour l'huile de colza, l'augmentation de la production (+ 330000 tonnes) et la diminution des utilisations humaines (- 430000 tonnes) ne permettent pas de compenser l'accroissement des utilisations en non-

\footnotetext{
${ }^{6}$ On doit remarquer que pour les céréales diverses, bien que la surface augmente légèrement, la production est en léger recul du fait d'un effet négatif des variations de prix sur le rendement.
} 
alimentaire (+ $3 \mathrm{Mt})$. L'UE est donc amenée à importer un supplément de 2,2 Mt de l'extérieur qui proviendraient principalement, selon cette simulation, de Chine, d'Afrique-Asie et de la zone " autres ». Pour les tourteaux, alors que la production augmente de 550000 tonnes, les utilisations en alimentation animale ne progressent que de 218000 tonnes avec un remplacement partiel du tourteau de soja par des tourteaux de colza et de tournesol devenus plus compétitifs. Les déficits de l'UE diminuent de 150000 tonnes pour le colza et de 250000 tonnes pour le soja. Au contraire, il s'aggrave légèrement pour le tournesol et le palmiste.

En pourcentage, on voit la très forte évolution du solde de l'UE en huile de colza. Alors qu'on avait en 2002 un excédent de l'ordre de l'ordre de 270000 tonnes, on passe à un déficit de près de $2 \mathrm{Mt}$. Le déficit augmente également en huiles de tournesol (+18\%), de palme
(+ $3 \%)$ et de palmiste $(+2,7 \%)$. En huile de soja, c'est l'« excédent » de 900000 tonnes $\mathrm{d}^{\prime}$ 'huiles provenant de graines locales ou importées qui est réduit de 6,7\%. Ces évolutions du commerce extérieur des différentes huiles sont fortement liées aux variations de production et surtout de demandes alimentaires. Ainsi, au niveau de la consommation humaine, alors que I'huile de colza recule de $20 \%$, celle de tournesol progresse de 7,4\% et celles de soja et palme de 3,6 à 3,7\%. En ce qui concerne les tourteaux, les utilisations progressent de $6 \%$ pour le colza, de $3 \%$ pour le tournesol et reculent de $0,8 \%$ pour le soja. Alors que le déficit en tourteaux de soja est réduit de 1,4\%, celui en tournesol progresse de 3,3\% (de 1,4 Mt à 1,45). Pour le colza, on partait d'une situation où I'UE présentait un déficit de 343000 tonnes et on arrive à une situation où le déficit est ramené à 193000 tonnes.

Tableau 7. Évolution des productions mondiales de graines de colza (\% et $1000 \mathrm{t}$ ).

\begin{tabular}{|lcccc|}
\hline & $\begin{array}{c}\text { Productions } \\
\text { initiales }\end{array}$ & $\begin{array}{c}\text { Variations relatives } \\
(\%)\end{array}$ & $\begin{array}{c}\text { Productions } \\
\text { finales }\end{array}$ & $\begin{array}{c}\text { Variations } \\
\text { absolues }\end{array}$ \\
\hline UE & 9286 & 8,8 & 10100 & 814 \\
AFR_ASI & 4081 & 2,9 & 4198 & 117 \\
USA & 706 & 0,0 & 706 & 0 \\
CHINE & 10552 & 0,8 & 10633 & 81 \\
AUTRES & 7834 & 4,9 & 8219 & 385 \\
Monde & 32459 & 4,3 & 33857 & 1398 \\
\hline
\end{tabular}

Unités : $1000 \mathrm{t}$ et \%. Remarque : les zones Argentine-Brésil et Asie du Sud-Est n'apparaissent pas dans ce tableau et dans les suivants dans la mesure où ils n'ont aucune production de colza.

Tableau 8. Évolution des productions mondiales d'huiles et tourteaux de colza (\% et $1000 \mathrm{t}$ ).

\begin{tabular}{|c|c|c|c|c|c|c|c|c|}
\hline & \multicolumn{2}{|c|}{$\begin{array}{c}\text { Variation relative } \\
(\%)\end{array}$} & \multicolumn{2}{|c|}{$\begin{array}{l}\text { Productions } \\
\text { initiales }\end{array}$} & \multicolumn{2}{|c|}{$\begin{array}{c}\text { Productions } \\
\text { finales }\end{array}$} & \multicolumn{2}{|c|}{$\begin{array}{c}\text { Variations } \\
\text { absolues }\end{array}$} \\
\hline & Huiles & Tourteaux & Huiles & Tourteaux & Huiles & Tourteaux & Huiles & Tourteaux \\
\hline UE & 9,3 & 9,3 & 3560 & 5180 & 3892 & 5662 & 332 & 482 \\
\hline AFR_ASI & 2,9 & 2,9 & 1415 & 2608 & 1456 & 2684 & 41 & 76 \\
\hline USA & 0,0 & 0,0 & 223 & 352 & 223 & 352 & 0 & 0 \\
\hline CHINE & 0,8 & 0,8 & 3538 & 6325 & 3567 & 6377 & 29 & 52 \\
\hline AUTRES & 5,1 & 5,1 & 3085 & 4341 & 3244 & 4564 & 158 & 223 \\
\hline Monde & 4,7 & 4,4 & 11821 & 18806 & 12381 & 19640 & 560 & 834 \\
\hline
\end{tabular}

Unités : 1000 t et $\%$.

Tableau 9. Évolution des consommations mondiales d'huiles et tourteaux de colza (\% et 1000 t).

\begin{tabular}{|lrcrcrrrr|}
\hline & \multicolumn{2}{c}{$\begin{array}{c}\text { Variations relatives } \\
\text { (\%) }\end{array}$} & \multicolumn{2}{c}{$\begin{array}{c}\text { Consommations } \\
\text { initiales }\end{array}$} & \multicolumn{2}{c|}{$\begin{array}{c}\text { Consommations } \\
\text { finales }\end{array}$} & Variations absolues \\
\cline { 2 - 9 } & Huiles & Tourteaux & Huiles & Tourteaux & Huiles & Tourteaux & Huiles & Tourteaux \\
\hline UE & 77,2 & 6,0 & 3330 & 5535 & 5900 & 5868 & 2570 & 333 \\
AFR_ASI & $-27,3$ & 6,0 & 1531 & 2180 & 1113 & 2310 & -418 & 130 \\
USA & $-22,2$ & 0,0 & 581 & 1240 & 452 & 1240 & -129 & 0 \\
CHINE & $-25,6$ & 2,5 & 3646 & 6123 & 2712 & 6274 & -934 & 151 \\
AUTRES & $-18,7$ & 5,9 & 2834 & 3736 & 2305 & 3955 & -529 & 219 \\
Monde & 4,7 & 4,4 & 11922 & 18814 & 12482 & 19648 & 560 & 834 \\
\hline
\end{tabular}

Unités : \% et 1000 tonnes.
Les évolutions sur le marché mondial du colza Après avoir examiné les principales implications du choc sur les productions, utilisations et solde commercial de l'UE, il convient d'examiner les principaux effets calculés concernant le marché mondial du colza. Au niveau des graines de colza (tableau 7), la production mondiale augmente de 1,4 Mt (+ 4,3\%), dont I'essentiel dans I'UE: +810000 tonnes $(+8,8 \%)$, l'essentiel du reste dans la zone " autres" (qui inclut le Canada et l'Australie) : +385000 tonnes $(+4,9 \%)$ et le solde dans Afrique-Asie (avec I'Inde et le Pakistan) et Chine.

Les évolutions des productions d'huiles et tourteaux de colza (tableau 8) suivent directement celles des évolutions de graines.

Au niveau des consommations totales d'huile de colza (homme, animal et industrie), les utilisations dans I'UE augmentent de $77 \%$ (soit $+2,57 \mathrm{Mt}$ ), et reculent de façon plus ou moins importante dans toutes les autres zones où cette huile est présente (tableau 9) en raison du renchérissement de son prix par rapport aux autres huiles (de - $19 \%$ à - $27 \%$ ). Le tourteau de colza, devenu plus compétitif, augmente de consommation à la fois dans I'UE (+ 333000 tonnes) et dans les autres zones où il était déjà consommé (Afrique et Asie du Sud avec I'Inde notamment, Chine et « autres »).

Au niveau des soldes mondiaux en huile, on a des changements très importants. L'UE qui avait un excédent de 272000 tonnes en huile de colza, voit son déficit passer à 1,97 Mt. Cette dégradation du solde commercial de 2,24 Mt est couverte par un développement des importations en provenance de la Chine (qui passe d'un déficit à un excédent de 855000 tonnes), des « autres» (+ 688000 tonnes), qui augmentent fortement leur excèdent, de l'AfriqueAsie (+ 459000 tonnes, qui passe d'un déficit de 60000 tonnes à un net excédent), enfin des États-Unis dont le déficit (essentiellement par rapport au Canada) est ramené de 372000 tonnes à 243000 tonnes. Pour les tourteaux, les variations sont moins importantes en tonnage et pourcentage. La diminution du déficit de l'UE en tourteaux de colza de 150000 tonnes, se traduit par une diminution des importations en provenance de la Chine $(-99000$ tonnes) et de I'Afrique-Asie (Inde) : - 54000 tonnes.

\section{Conclusions}

L'hypothèse de transmission totale des variations de prix de l'huile de colza conduirait donc à une situation où, pour répondre à une demande supplémentaire exogène $d^{\prime}$ huile de colza de 3 Mt dans I'UE, la production mondiale de cette graine augmenterait de seulement 1,4 Mt dans le monde, soit 580000 tonnes en équivalent huile. Globalement, le 
solde de l'UE en graines, huiles et tourteaux de colza se dégrade donc fortement et les pays tiers interviennent de plus en plus dans l'approvisionnement communautaire. Ces résultats vont bien dans le sens de ce que l'on a observé au cours des dernières années, mais sousestiment l'ampleur de certaines évolutions notamment en termes d'augmentation des surfaces dans l'UE et niveaux de rapports de prix entre les différentes huiles. Pour représenter plus précisément les phénomènes réels, on a recouru dans une seconde simulation, toujours avec le même choc exogène de $3 \mathrm{Mt}$, à une modélisation classique qui est celle des transmissions partielles de prix. Celle-ci permet de prendre en compte le fait que les zones du monde qui ont une consommation intérieure essentiellement basée sur leurs ressources locales sont faiblement influencées par les évolutions des prix « mondiaux » du produit considéré, alors que celles qui sont fortement impliquées dans le commerce mondial (en tant qu'exportateurs ou importateurs) perçoivent beaucoup plus fortement les signaux du marché mondial dès lors qu'elles ont des politiques plus « libérales». Cette forme de modélisation permet aussi de mieux représenter une situation de moyen terme consécutive au déclenchement d'un choc exogène dans une phase précédant la réalisation de tous les mécanismes d'ajustement, notamment entre les prix des différentes zones. Enfin, cette modélisation permet de façon qualitative de prendre en compte le fait que les transformateurs situés dans une zone géographique ont généralement une certaine préférence pour les produits locaux.

\section{Effets du choc avec transmission partielle des prix}

Dans ce scénario, les variations de prix de I'huile de colza se transmettent pour $50 \%$ en Chine et Afrique-Asie, et pour $100 \%$ pour toutes les autres zones et produits. Pour la Chine et Afrique-Asie, le coefficient plus faible relatif à I'huile de colza correspond au fait que ces deux zones sont d'importants producteurs de cette huile (respectivement 3,5 et 1,4 Mt sur un total mondial hors UE de 8,2 Mt), mais que celle-ci est totalement destinée à leurs marchés intérieurs qui sont en partie déconnectés des prix mondiaux. Par contre, la zone « autres», qui comprend le Canada et l'Australie (production de 3,1 Mt), est fortement connectée au marché mondial sur lequel ces pays exportent une part importante de leur production de graine qui s'ajuste régulièrement en fonction des prix mondiaux.

\section{Les évolutions de prix dans I'UE}

Au niveau de I'UE, les principales différences par rapport au scénario précédent portent sur
I'évolution des prix de l'huile de colza. Alors qu'en cas de transmission totale, le prix augmente $18,9 \%$, il augmente de $24,2 \%$ en cas de transmission partielle. Ceci s'explique par le fait que la Chine et la zone " autres » ne perçoivent que partiellement le signal de la demande européenne et donc augmentent moins fortement leur production et trituration qu'en cas de transmission totale. La hausse de prix dans I'UE pour l'huile de colza est donc plus forte que dans le cas précédent pour rationner davantage la demande alimentaire au bénéfice du non alimentaire Pour les tourteaux de colza, la baisse est légèrement plus forte $(5,8 \%$ contre $5,2 \%)$ que dans le cas précédent. Globalement, la hausse du prix de la graine de colza dans I'UE est de 15,9\% contre $12,3 \%$, ce qui lui permet d'augmenter davantage ces surfaces.

\section{Les évolutions de production dans I'UE}

Compte tenu de la hausse plus forte du prix de la graine de colza, la production de I'UE augmente de 1,06 Mt au lieu de 814000 tonnes et les productions en céréales reculent plus fortement, notamment pour le blé (tableau 10).

\section{Les évolutions des productions et bilans mondiaux}

Avec transmission partielle des prix, la production mondiale de graines de colza (tableau 11) augmente de 1,7 million de tonnes, contre 1,4 dans le cas précédent, mais se trouve beaucoup plus localisée dans I'UE et dans la zone « autres» (Canada, Australie). Ceci revient à dire que la Chine et I'Afrique-Asie où les prix des graines augmentent moins que dans I'UE et en « autres » ajustent moins leur production à la demande supplémentaire de I'UE qui doit être satisfaite davantage par sa propre production et par celle de la zone « autres » (résultats par zone non détaillés).

Compte tenu d'un rationnement plus fort de la demande alimentaire de l'UE en huile de colza, en cas de transmission partielle, l'augmentation du déficit est de « seulement »1,9 Mt au lieu de 2,2 dans le cas de la transmission totale. II est couvert pour plus de moitié par les « autres » (Canada, Australie), mais on observe malgré tout (tableau 12) des augmentations des importations en provenance de Chine et d'Afrique-Asie, moins importantes toutefois que dans le cas de transmission totale. Compte tenu de la plus forte augmentation du prix de I'huile de colza, les États-Unis diminuent plus fortement leurs importations en provenance des « autres » (Canada).

Pour les tourteaux de colza (tableau 13), la progression de demande mondiale, du fait de prix en plus forte baisse est légèrement supérieure à celle en cas de transmission totale (984 000 contre 834000 tonnes). Le déficit de I'UE diminue de 234000 tonnes alors que les

Tableau 10. Évolution des productions de céréales et graines oléagineuses dans I'Union européenne avec transmission totale et partielle.

\begin{tabular}{|lrcccc|}
\hline & \multicolumn{2}{c}{ Transmission totale } & \multicolumn{2}{c|}{ Transmission partielle } \\
\cline { 2 - 6 } & $\begin{array}{l}\text { Niveaux } \\
\text { initiaux }\end{array}$ & $\begin{array}{c}\text { Variations relatives } \\
(\%)\end{array}$ & $\begin{array}{c}\text { Différences } \\
\text { Variations relatives } \\
(\%)\end{array}$ & Différences \\
\hline BLE & 103894 & $-1,3$ & -1373 & $-1,7$ & -1724 \\
CERDI & 18211 & $-1,1$ & -196 & $-1,4$ & -246 \\
MAIS & 39450 & $-1,0$ & -412 & $-1,3$ & -518 \\
ORGE & 47962 & $-1,4$ & -656 & $-1,7$ & -824 \\
COLZA & 9286 & 8,8 & 814 & 11,5 & 1063 \\
SOJA & 814 & 0,81 & 7 & 0,69 & 6 \\
TOURNESOL & 2753 & 4,38 & 121 & 4,44 & 122 \\
Total * & 224662 & $-0,76$ & -1703 & $-0,95$ & -2130 \\
\hline
\end{tabular}

Unités : 1000 tonnes; * y compris riz.

Tableau 11. Évolution des productions mondiales de colza avec transmission totale et partielle.

\begin{tabular}{|lccccr|}
\hline & \multicolumn{2}{c}{ Transmission totale } & \multicolumn{2}{c|}{ Transmission partielle } \\
\hline & Niveaux initiaux & $\begin{array}{c}\text { Variations } \\
\text { relatives (\%) }\end{array}$ & Différences & $\begin{array}{c}\text { Variations } \\
\text { relatives (\%) }\end{array}$ & Différences \\
\hline UE & 9286 & 8,8 & 814 & 11,5 & 1063 \\
AFR_ASI & 4081 & 2,9 & 117 & 1,2 & 49 \\
USA & 706 & 0,0 & 0 & 0,0 & 0 \\
CHINE & 10552 & 0,8 & 81 & 0,3 & 33 \\
AUTRES & 7834 & 4,9 & 385 & 6,6 & 519 \\
Monde & 32459 & 4,3 & 1398 & 5,1 & 1664 \\
\hline
\end{tabular}

Unités : 1000 tonnes. 
Tableau 12. Variations des bilans huile de colza mondiaux avec transmission totale et partielle.

\begin{tabular}{|lcccccc|}
\hline & \multicolumn{3}{c}{ Transmission totale } & \multicolumn{3}{c|}{ Transmission partielle } \\
& Productions & Consommations & Échanges & Productions Consommations & Échanges \\
\hline UE & 332 & 2570 & -2239 & 433 & 2323 & -1890 \\
AFR_ASI & 41 & -418 & 459 & 17 & -152 & 169 \\
USA & 0 & -129 & 129 & 0 & -219 & 219 \\
CHINE & 29 & -934 & 963 & 12 & -437 & 449 \\
AUTRES & 158 & -529 & 688 & 214 & -839 & 1053 \\
Monde & 560 & 560 & 0 & 676 & 676 & 0 \\
\hline
\end{tabular}

Unités : 1000 tonnes.

Tableau 13. Variations des bilans tourteau de colza mondiaux avec transmission totale et partielle.

\begin{tabular}{|lcccccc|}
\hline & \multicolumn{3}{c}{ Transmission totale } & \multicolumn{3}{c|}{ Transmission partielle } \\
\cline { 2 - 6 } & Productions & Consommations & Échanges & Productions & Consommations & Échanges \\
\hline UE & 482 & 333 & 150 & 630 & 396 & 234 \\
AFR_ASI & 76 & 130 & -54 & 32 & 152 & -120 \\
CHINE & 52 & 151 & -99 & 21 & 177 & -156 \\
AUTRES & 223 & 219 & 4 & 301 & 259 & 42 \\
Monde & 834 & 834 & 0 & 984 & 984 & 0 \\
\hline
\end{tabular}

Unités : 1000 tonnes.

quantités exportées de Chine et de la zone « autres» sont en sensible recul ( -156000 tonnes et - 120000 tonnes) du fait d'une nette progression de leurs utilisations intérieures.

\section{Conclusions}

Avec l'hypothèse de transmission partielle des prix, on a une situation où les prix de l'huile de colza (et donc de cette graine) se trouvent partiellement déconnectés entre les différentes zones du monde, ce qui induit une plus forte progression de la production d'oléagineux au sein de I'UE et une moindre modification des bilans internationaux. Cependant, dans ce cas comme dans le précédent, c'est l'ensemble des grandes cultures mondiales (céréales et oléagineux) qui se trouve affecté, en termes de productions et d'utilisations, par un choc initialement limité à l'UE et à la seule huile de colza. Même si, comme on I'a indiqué précédemment, des éléments autres que le développement du biodiesel ont joué au cours des dernières années sur l'évolution des marchés communautaires et internationaux des huiles et graines oléagineuses, il est intéressant de comparer les résultats de ce second scénario avec les évolutions effectivement observées au cours de la période 2002-2005, sachant que les résultats du modèle concernent les seuls 15 anciens pays de I'UE alors que 10 nouveaux membres, pour certains gros producteurs de colza, ont rejoint l'UE le $1^{\text {er }}$ mai 2004 modifiant ainsi les équilibres de marché. En l'absence de données précises sur les productions de biodiesel dans I'UE à 15 en 2005, on a retenu une estimation de 2,4 Mt qui correspond à une progression d'environ 1,5 Mt de la consomma- tion d'huile en non-alimentaire. Ce chiffre est à mettre en relation avec le choc simulé de $3 \mathrm{Mt}$. En termes de rapport de prix du colza par rapport au soja, pour les huiles on est passé de 1,067 en 2002 à 1,33 en 2005, soit une progression du ratio de $25 \%$. Dans la seconde simulation, l'augmentation calculée est de $12,5 \%$. Pour les tourteaux, le ratio réel est passé de 0,67 en 2002 à 0,62 en 2005, soit une diminution de $7 \%$. Le modèle indique une diminution 5,4\%. En termes de superficies cultivées en colza, pour les 15 anciens membres de l'UE, les surfaces sont passées de 3,1 Mha en 2002 à 3,47 en 2005, soit une progression de $12 \%$ alors que le modèle fournit un chiffre très voisin de $11,5 \%$. Au niveau des récoltes de graines, on est passé de 9,5 Mt à 12,2 Mt (soit $+28 \%$ ) grâce à de très bons rendements au cours de cette année. Cet aspect « conjoncturel $\gg$ de rendement $n^{\prime}$ est pas pris en compte dans le modèle.

Globalement, il apparaît donc que le modèle Oléosim, dans sa structure actuelle, permet bien de refléter les principales évolutions de marché dues au développement de la demande non alimentaire en huiles végétales. Cependant, avec une simulation de choc de demande environ deux fois plus important que le choc réel, on arrive à une même progression de surface dans I'UE que celle effectivement observée, à une baisse du ratio de prix des tourteaux proche de la réalité, mais à une progression du ratio de prix des huiles nettement plus faible. Cette différence pourrait principalement s'expliquer par la baisse des productions de graines en 2005 de l'Australie, de la Pologne, de la République Tchèque et, dans une moindre mesure, de la Chine, et par la forte demande en colza durant la campagne en cours de pays tels que le Mexique, le Japon et surtout la Chine. Ces divers éléments expliqueraient à côté de la demande non alimentaire dans I'UE une part importante de l'augmentation de ratio des huiles de colza et de soja. Le fait que la Chine soit en passe d'importer environ 1,2 Mt de graines de colza canadiennes en 2005/2006 contre seulement 0,3 Mt durant la campagne précédente montre bien qu'il y a seulement, au niveau de certaines zones hors $\mathrm{UE}$, une transmission partielle des effets de variations des prix communautaires, le coefficient retenu de $50 \%$ étant probablement trop élevé dans la situation actuelle. Le dernier élément de déconnexion entre le marché communautaire du colza et le marché mondial tient enfin au fait que, pour des raisons liées au caractère OGM des productions de "canola » canadiennes, l'UE s'est mise depuis des années totalement en marge de cette origine pour son approvisionnement en graines, tourteaux et huiles, alors que ce pays est de très loin le principal exportateur mondial et celui où la production tend à croître le plus rapidement. De façon générale, on peut s'interroger sur la durabilité d'un différentiel des prix communautaires entre huile de colza et autres huiles, dans la mesure où il est en partie expliqué par l'exclusion «volontaire » des potentialités offertes par le Canada.

Les simulations présentées permettent donc d'aborder l'analyse de l'impact économique possible d'un certain développement de la production de biodiesel dans I'UE, cependant ces résultats reposent sur diverses d'hypothèses simplificatrices qui doivent être discutées et impliquent des prolongements au travail de recherche actuel.

\section{Conclusion}

Les résultats des simulations présentés ci-dessus confirment en premier lieu le rôle du non alimentaire dans les évolutions du marché international et des bilans communautaires du colza présentés dans la première partie de cet article, notamment en ce qui concerne l'évolution des prix et rapports de prix et la tendance de I'UE à utiliser de plus en plus les ajustements de son commerce extérieur en graines et huiles de colza pour satisfaire l'accroissement de ses besoins intérieurs en huiles. Cependant, ils montrent également que les autres facteurs de nature conjoncturelle influençant traditionnellement ces marchés continuent à avoir un rôle très important (notamment les effets de rendement dans I'UE et certains pays tiers). De façon plus générale, il convient d'introduire dans l'analyse, et donc dans les prochaines simulations à réaliser avec le modèle Oléosim, quatre éléments principaux. 
Tout d'abord, si au niveau de l'UE le développement du biodiesel a été très important et va sûrement se poursuivre, dans I'UE et dans de nombreux pays tiers la production de biocarburants à base d'éthanol provenant de céréales (essentiellement maïs aux États-Unis) est également en forte croissance, ce qui a pour effet $d$ 'induire une plus grande fermeté des cours internationaux de ces céréales du fait de plus fortes utilisations à l'intérieur des diverses zones et donc globalement de moindres disponibilités à l'exportation. De ce point de vue, la prise en compte simultanée dans le modèle du développement de la demande non alimentaire en colza et en céréales, du fait de moindres effets de réallocations des surfaces entre ces cultures, devrait conduire à de plus fortes hausses de prix des céréales et oléagineux que ceux précédemment calculés, à moins de décisions politiques, notamment aux États-Unis et dans I'UE de remettre en culture certaines terres actuellement gelées. Par ailleurs, le développement de l'industrie de l'éthanol conduit à la mise sur le marché de quantités fortement croissantes de drèches qui viennent directement concurrencer les tourteaux et autres matières riches en protéines sur le marché de l'alimentation animale, directement dans les pays producteurs, mais aussi dans les autres par le biais de la mise en place de circuits d'exportation.

En second lieu, dans les simulations, on a retenu I'hypothèse d'un développement $d u$ biodiesel limité à I'UE. Or les conditions économiques et environnementales qui ont conduit à ce développement dans I'UE s'appliquent aussi dans de nombreux autres pays du monde, ce qui pourrait à terme conduire à des prélèvements sur le marché mondial "alimentaire » des huiles et corps gras végétaux très importants ayant naturellement des conséquences considérables en termes de commerce et de prix mondiaux. Ceci concerne en partie l'huile de soja aux États-Unis, mais également, et de façon encore plus forte en raison de sa très forte compétitivité prix, l'huile de palme dans les grands pays producteurs actuels.

En troisième lieu, on a retenu I'hypothèse que la demande communautaire pour le biodiesel, était actuellement - et continuerait à être essentiellement satisfaite par de l'huile de colza. Compte tenu des différentiels de prix très importants qui sont en train de s'instaurer entre les différents corps gras végétaux et ani- maux, on peut penser que les contraintes techniques qui privilégient actuellement cette huile seront plus ou moins rapidement levées par des efforts de recherche permettant une plus grande substitution au niveau du biodiesel entre huiles de colza, de soja, de tournesol et de palme, voire avec les graisses animales. II est clair qu'à ce niveau une forte demande mondiale non différenciée en corps gras végétaux et animaux aurait des conséquences très différentes de demandes « captives » spécifiques à certains produits dans les différents pays du monde. En tout état de cause, cette demande supplémentaire impliquerait bien toujours une hausse des prix de l'ensemble du complexe corps gras, mais devrait avoir des effets de distorsion de prix entre produits beaucoup plus limités.

Enfin, en ce qui concerne les réponses des différents pays en termes de surface aux hausses prévisibles de prix des huiles végétales et donc des graines, dans nos simulations les effets apparaissent comme relativement limités. On a en fait retenu des élasticités d'offre de moyen terme qui représentent les réponses des agriculteurs à des variations conjoncturelles de prix en terme d'affectation de leurs surfaces. II est clair que si de façon structurelle les prix des graines oléagineuses se revalorisent de façon notable par rapport à ceux des céréales, des ajustements structurels seront très probablement mis en place, notamment par le biais $d^{\prime}$ efforts de recherche et de développement qui permettraient aux oléagineux de revenir plus fréquemment dans les assolements. En tout état de cause, il restera la limite inhérente aux surfaces disponibles dans le monde pour I'ensemble des grandes cultures et la part incompressible de la demande à finalité alimentaire, aussi bien en céréales qu'en huiles végétales. La prise en compte de tous ces éléments dans une version plus complète du modèle Oléosim et la définition de nouveaux scénarios constitue une orientation du travail de recherche mené actuellement dans I'Unité d'économie de l'Inra de Rennes.

\section{RÉFÉRENCES}

1. OIL WORLD ANNUAL. ISTA Mielke. Hamburg : $\mathrm{GmbH}, 2005$.

2. OIL WORLD WEEKLY. ISTA Mielke. Hamburg: $\mathrm{GmbH}, 2005$.
3. EUROBSERV'ER. Le baromètre des biocarburants. Juin 2004.

4. PROLEA. Statistiques des oléagineux et protéagineux, 2004/2005. Paris. 2005.

5. ONIC/ONIOL. Les aides aux surfaces en France en 2005. décembre 2005 ; (Paris).

6. COMMISSION EUROPÉENNE. Livre vert sur la sécurité de l'approvisionnement en énergie. Bruxelles. Novembre 2000.

7. MINISTERE FRANÇAIS DE L'ECONOMIE ET DES FINANCES. Rapport de la France concernant la Directive 2003/30/CE visant à promouvoir I'utilisation des biocarburants. 2005.

8. PREVOT H, et al. Rapport pour Ministère Français de l'Économie et des Finances, Rapport sur l'optimisation du dispositif de soutien à la filière biocarburants. Septembre 2005.

9. COMMISSION EUROPÉENNE. Bio energy's role in the EU energy market, a view of developments until 2020. Avril 2004 ; (Bruxelles).

10. COMMISSION EUROPEENNE. Prospects for agricultural markets and income. mai 2005; (Bruxelles).

11. DRONNE Y, GAUTIER P, GOHIN A, LEVERT F, OLEOSIM. Un outil de modélisation du marché mondial des oléagineux : méthodologie et application à l'analyse de l'impact des aides nordaméricaines aux oléagineux. INRA SAE2. Rennes: Unité d'Économie, mai 2003.

12. GOHIN A, DRONNE Y, LEVERT F, DEBAR JC. Les instruments de politique agricole des ÉtatsUnis : analyse de leur impact sur la formation des prix mondiaux : quels préjudices pour les filières agricoles européennes, INRA SAE2. Rennes: Unité d'Économie, décembre 2005.

13. MORIN L, SURRY Y. Modélisation et estimation de la demande alimentaire d'huiles et graisses dans I'Union européenne. Cahiers d'Économie et Sociologie Rurales 1997 ; 45 : 21-60.

14. SURRY Y. The 'Constant Difference of Elasticities' function with applications to the EC animal feed sectors. / Agricul Econom 1993; 44(1): 110-25.

15. SULLIVAN J, RONINGEN V, LEETMA S, GRAY D. A 1989 Clobal Database for the Static World Policy Simulation Modeling Framework (SWOP. SIM). Staff Report AGES 9215. 1992.

16. VON TONGEREN F, VAN MEIIL H, SURRY Y. Global models applied to agricultural and trade policies: a review and assessment. Agric Econ $2001 ; 2$ : 149-71 